\title{
Dynamic Model and Fault Feature Research of Dual-Rotor System with Bearing Pedestal Looseness
}

\author{
Nanfei Wang, Hongzhi Xu, and Dongxiang Jiang \\ State Key Laboratory of Control and Simulation of Power System and Generation Equipment, Department of Thermal Engineering, \\ Tsinghua University, Beijing 100084, China
}

Correspondence should be addressed to Nanfei Wang; wnf14@mails.tsinghua.edu.cn

Received 28 February 2016; Accepted 4 May 2016

Academic Editor: Zhike Peng

Copyright (c) 2016 Nanfei Wang et al. This is an open access article distributed under the Creative Commons Attribution License, which permits unrestricted use, distribution, and reproduction in any medium, provided the original work is properly cited.

The paper presents a finite element model of dual-rotor system with pedestal looseness stemming from loosened bolts. Dynamic model including bearing pedestal looseness is established based on the dual-rotor test rig. Three-degree-of-freedom (DOF) planar rigid motion of loose bearing pedestal is fully considered and collision recovery coefficient is also introduced in the model. Based on the Timoshenko beam elements, using the finite element method, rigid body kinematics, and the Newmark- $\beta$ algorithm for numerical simulation, dynamic characteristics of the inner and outer rotors and the bearing pedestal plane rigid body motion under bearing pedestal looseness condition are studied. Meanwhile, the looseness experiments under two different speed combinations are carried out, and the experimental results are basically the same. The simulation results are compared with the experimental results, indicating that vibration displacement waveforms of loosened rotor have "clipping" phenomenon. When the bearing pedestal looseness fault occurs, the inner and outer rotors vibration spectrum not only contains the difference and sum frequency of the two rotors' fundamental frequency but also contains $2 X$ and $3 X$ component of rotor with loosened support, and so forth; low frequency spectrum is more, containing dividing component, and so forth; the rotor displacement spectrums also contain fewer combination frequency components, and so forth; when one side of the inner rotor bearing pedestal is loosened, the inner rotor axis trajectory is drawn into similar-ellipse shape.

\section{Introduction}

Pedestal looseness is one of the common faults in rotating machinery, which will lead to the severe vibration of the whole system. Particularly, when the looseness fault is serious, it may cause the failure of equipment and even some catastrophic accidents. Looseness fault is usually caused by the poor quality of installation or long-term vibration. Under the action of the imbalance force, the rotor system with pedestal looseness will have a periodic beating. It is difficult to detect pedestal looseness fault due to the complicated vibration phenomenon. Therefore, it is significant to diagnose the existence and severity of pedestal looseness for the safe and reliable operation of rotating machinery.

Aimed at the dynamics and fault diagnosis of rotor systems with pedestal looseness, a large number of studies have been carried out and many results have been achieved.
Goldman and Muszynska [1] established the bilinear model of a rotating machine with one loose pedestal. The synchronous and subsynchronous fractional components of the response were demonstrated and conform to the experimental results. Subsequently [2], they discussed the chaotic behaviour of the system based on the bilinear model. Chu and Tang [3] investigated the vibration characteristics of a rotor-bearing system with pedestal looseness by establishing a nonlinear mathematical model. Stability of periodic solutions was analysed by means of the shooting method and the Floquet theory. Ma et al. [4] presented a finite element model of a rotor system with pedestal looseness stemming from a loosened bolt, and the effects of the looseness variables (foundation stiffness, stiffness of nonloosened bolts, rotating speed, and looseness clearance) on its dynamic characteristics were investigated. When the rotating speed and imbalance of rotors varied, periodic, quasiperiodic, and chaotic motions could be detected 
and three kinds of routes to or out of chaos were observed. In some cases, pedestal looseness could cause $1 X / 2$ fractional harmonic and multiple harmonic motions of rotor-bearing of systems [5]. Ji and $\mathrm{Zu}$ [6] analysed the free and forced vibrations of a nonlinear bearing system to illustrate the nonlinear effect on the free and forced vibrations of the system by the method of multiple scales. Ma et al. [7] built a mechanical model of looseness of fastening bolt on the bearing pedestal and analysed the dynamic characteristics of rotor by adopting the nonlinear oil-film model developed by Adiletta. The results illustrate that system motion state changes frequently with the increase of the rotating speed. Lu et al. [8] set up the model of seven-degree-of-freedom rotor system including a pair of ball bearings with pedestal looseness at one end by utilizing Newton's second law and analysed the stability of the model based on the known harmonic solution by the Floquet theory. Qin et al. [9] investigated the bolt loosening at the rotating joint interface and its influence on the rotor dynamics, and the nonlinear FE simulations were performed to calculate the time-varying stiffness at the joint interface with bolt loosening. Fault diagnosis of rotor system with pedestal looseness has been widely considered utilizing different analytical methods, such as wavelet analysis, Hilbert-Huang transform, and genetic algorithm [10-13].

Recently, many scholars studied pedestal looseness of rotor system by using finite element method which may take into account many factors, such as mass, moment inertia, internal damping, bending, and torsion vibration coupling effects. Ma et al. [14] investigated the nonlinear vibration characteristics of a rotor system with pedestal looseness fault under different loading conditions. Wang and Chen [15] set up a whole rotor-support-casing model with looseness fault for certain type of turbofan aeroengine. The casing acceleration response characteristics were analysed. Behzad and Asayesh [16] proposed a finite element code for studying the effects of loose rotating disks on the rotor-bearing systems' response. The developed finite element model can numerically calculate the response of rotors with any number of loose discs at any location with isotropic or orthotropic supports. Wang et al. [17] introduced two looseness fault models, and the mechanism of the asynchronous vibration response phenomenon caused by the looseness fault in the whole aeroengine vibration system was analysed by numerical integration methods.

Many models with looseness fault involved in previous researches focus on simple Jeffcott rotor system using the lumped mass model and finite element model without regard to the dual-rotor structure of real aeroengine. Meanwhile, translation motion of pedestal is just considered in traditional pedestal looseness models. In fact, pedestal not only does plane motion but also does rotary motion around the axis in radial plane when pedestal looseness occurs, which will cause the pedestal's collision with the foundation and bolts. In our study, a nonlinear finite element model of the dualrotors-bearing-foundation system with pedestal looseness is established. The research results may give deep insight into looseness mechanism.

The motivation of the paper is to detect the dynamic response under pedestal looseness status of the dual-rotor system, which is widely used in aircraft engines and other real rotating machines. In order to investigate the dynamic characteristics of pedestal looseness, a dual-rotor system dynamic model is established based on dual-rotor system test rig and one-dimensional finite element model of the dual-rotor system, in which loosened pedestal is considered a rigid body doing plane motion and pedestal's collision with foundation and bolts is also considered. Furthermore, the dynamic characteristics are verified by comparing them with the results measured from experiments.

\section{Mathematical Model of Pedestal Looseness Fault}

2.1. Introduction to the Dual-Rotor Experiment Rig. Figures 1(a) and 1(b) show the dual-rotor system test rig and structure diagram, respectively. The inner rotor (1) passing through the outer rotor (2) is connected to a flexible coupling that is driven by a high-speed motor; the outer rotor is driven by a high-speed motor with a belt. Every bearing is supported on one pedestal (13), which is fixed on the foundation by several bolts. The bearing pedestal and membrane coupling (12) can reduce the influence of transverse force due to belt driven. The inner rotor and outer rotor are, respectively, installed with wheel disks to simulate the compressor and turbine load. Both ends of inner rotor are supported by deep groove ball bearing (4) and roller bearing (5), respectively; one end of outer rotor is supported by deep groove ball bearing (6), and the other end is supported on the inner rotor by means of squirrel cage elastic support and roller bearing (7). Two wheel disks $(8,9)$ are mounted on the inner rotor, and there are three wheel disks $(10,11,12)$ installed on the outer rotor. The elastic support is installed on the wheel disk, the number of which is (11).

One-dimensional finite element model of dual-rotor system (excluding pedestal) is built based on Timoshenko beam element, as presented in Figure 2, which is composed of the inner rotor (node 1 to node 13 ) and the outer rotor (node 14 to node 20). There are a total of four bearings in the model, which are, respectively, located at node 1 , node 14 , node 9 , and node 13 , where node 1 and node 13 denote the intershaft bearings. The disks, located in node 16 , node 18 , node 3 , and node 11 , represent the concentrations of highpressure compressor disks, high-pressure turbine disks, lowpressure compressor disks, and low-pressure turbine disks, respectively.

2.2. The Determination of Dual-Rotor Experimental Test Normal/Fault State. In the initial period of dual-rotor experimental rig operation, the normal status should be firstly determined, and the specific practices are as follows: when the fundamental frequencies $X_{1}$ and $X_{2}$ observed from the test and control system written in LABVIEW are most prominent $\left(X_{1}\right.$ represents the fundamental of inner rotor and $X_{2}$ represents the fundamental of outer rotor), it is considered to be the normal status, as shown in Figure 3(a). If the deviation with the state illustrated in Figure 3(a) is larger, the experimental rig will be adjusted to conform to the normal status. It should be noted that the spectrum also contains 


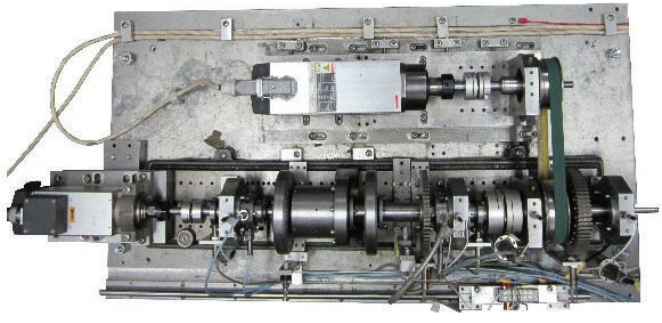

(a) Dual-rotor system test rig

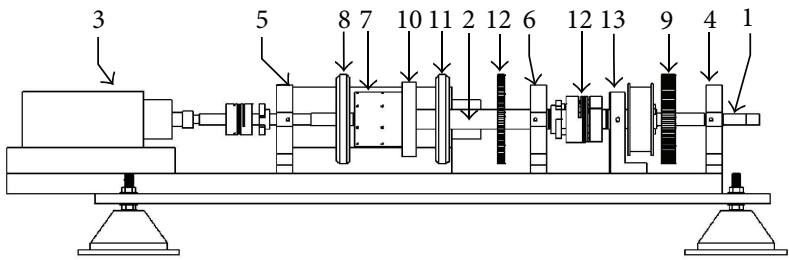

(b) Structure diagram of dual-rotor experiment setup

FIGURE 1: Dual-rotor system test rig and structure diagram.

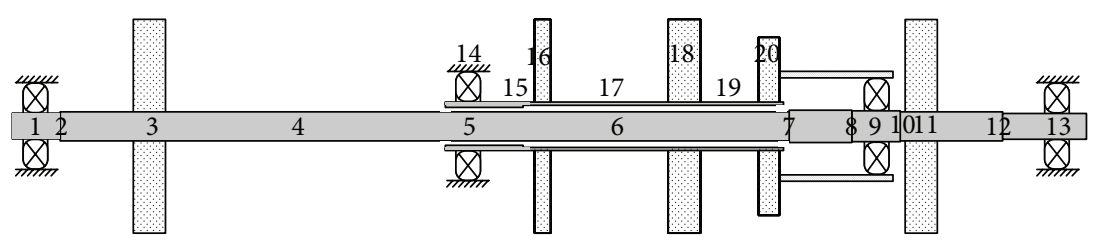

FIGURE 2: The node map of one-dimensional finite element model of dual-rotor system.

other frequency components with relatively small amplitude under normal status, just because of inevitable noise and mechanical assembly error.

When the pedestal looseness fault simulation is carried out in the dual-rotor experimental rig, it can be clearly seen that the spectrum diagram shown in Figure 3(b) not only contains fundamental frequencies $X_{1}$ and $X_{2}$, the sum frequency, and difference frequency of two rotors' fundamental frequencies but also contains some superharmonic components (such as $2 X$ and $3 X$ ). Comparing with the spectrum under normal status, the frequency components circled in red ellipse are presented in Figure 3(b) due to pedestal looseness.

2.3. The Description of Looseness Fault. The dual-rotor test rig is employed in the study as shown in Figure 1, and the bearing is mounted on the pedestal which is installed on the foundation with bolts and disk springs. The analysis for the case of unilateral looseness of pedestal which is used to support the roller bearing of inner rotor is carried out in this paper, as indicated in Figure 4. The position limit block is applied to restrain the axis motion of bearing pedestal. The force exerted on the bearing because of rotor vibration is transferred to the pedestal when fastening bolts are loose, resulting in the motion of pedestal. In general, the stiffness and strength of pedestal are high, so the pedestal is considered a rigid body to analyse. Nevertheless, in the local area of the collision occurring between the pedestal and foundation, the contact stiffness should be introduced to conform to the actual situation. Collision and friction exist between the pedestal and foundation as well as bolts, so the trajectory is relatively complex. As for the dual-rotor system test rig in the research, the fastening bolts on one side of the pedestal are loosened to simulate the pedestal looseness. The motion of pedestal in rotor axis direction (defined as $Z$-direction) is ignored, and only the translational motion and rotation around the $Z$-axis in the $X Y$ plane are considered.

2.4. The Dynamic Model of Pedestal Looseness. It is assumed that the pedestal does rigid motion in $X Y$ plane. The bolt bending in $X$-direction is taken into account, and the friction that existed between the pedestal and foundation in $X$ direction is neglected; the stretching of bolt and the collision that occurred between the pedestal and foundation in $Y$ direction is considered. The pedestal can be treated to do rigid motion including collision in the dynamic model, as shown in Figure 5.

It can be seen from Figure 5 that $\mathrm{A}$ and $\mathrm{B}$ denote the left edge and right edge of the pedestal; $C$ represents the gravity centre of the pedestal; $\mathrm{D}$ denotes the centre of bearing's outer ring; E represents the centre of rotor mounted on the bearing's inner ring. The bolt fastenings that existed between the left and right edges of pedestal and foundation are simplified as spring connection. Similarly, the fastenings that existed between the centre of bearing's outer ring and rotor centre are also simplified as spring connections in $X$-direction and $Y$-direction.

The fastening bolts are stretched when the pedestal is separated from the foundation to move upward, and $K_{2 y m}$ and $K_{3 y m}$ are corresponding spring stiffness, respectively. The collision area can be seen as elastic collision involving greater contact stiffness when the pedestal moves downward and collides with the foundation, and the contact stiffness between the left and right edges and foundation is $K_{2 y d}$ and $K_{3 y d}$, respectively. Deformation lengths of the spring are, respectively, defined as $d_{2 y}$ and $d_{3 y}$ in $Y$-direction; their values are greater than 0 , which means that the pedestal is separated from the foundation and moves upward; their 

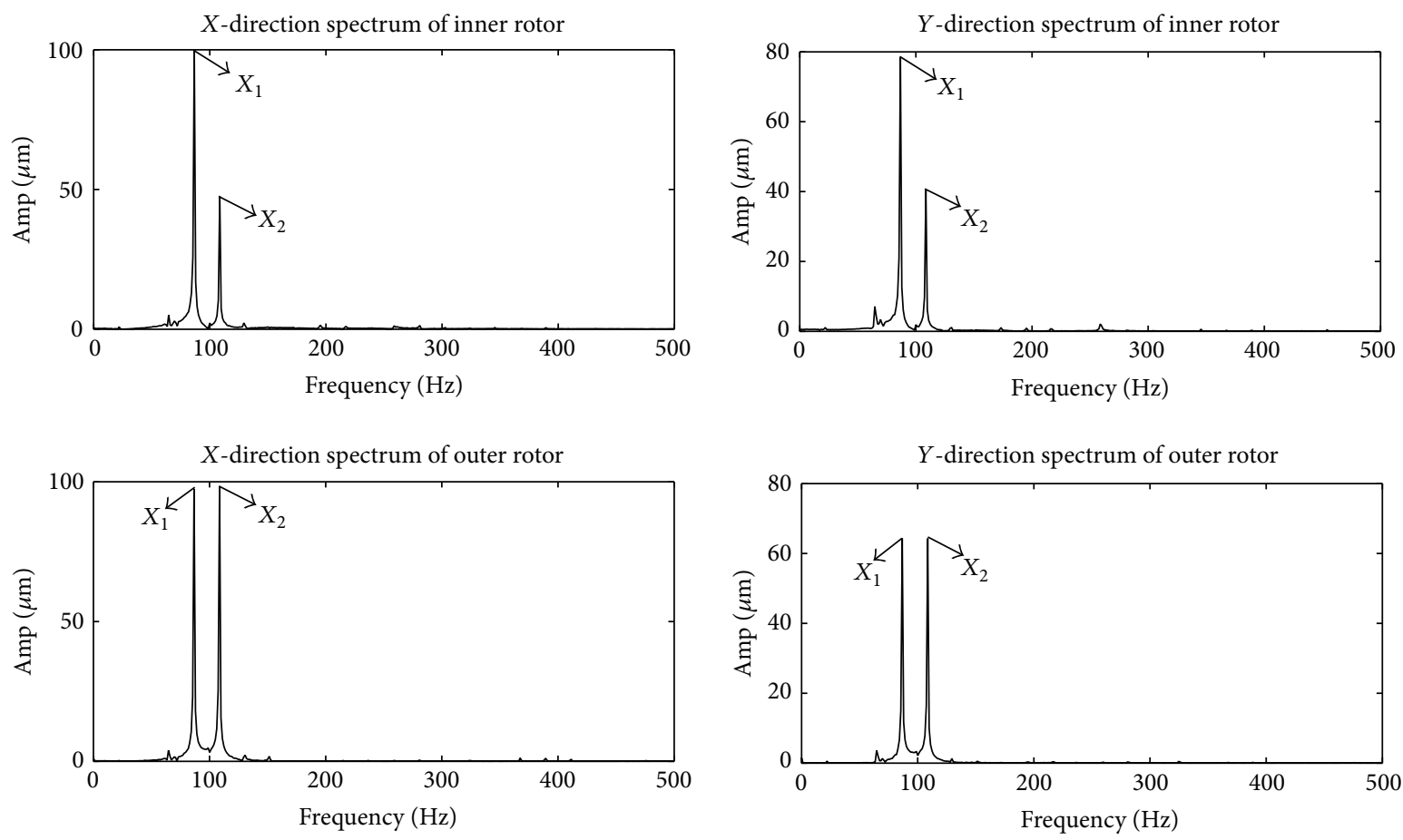

(a) The spectrum under normal status
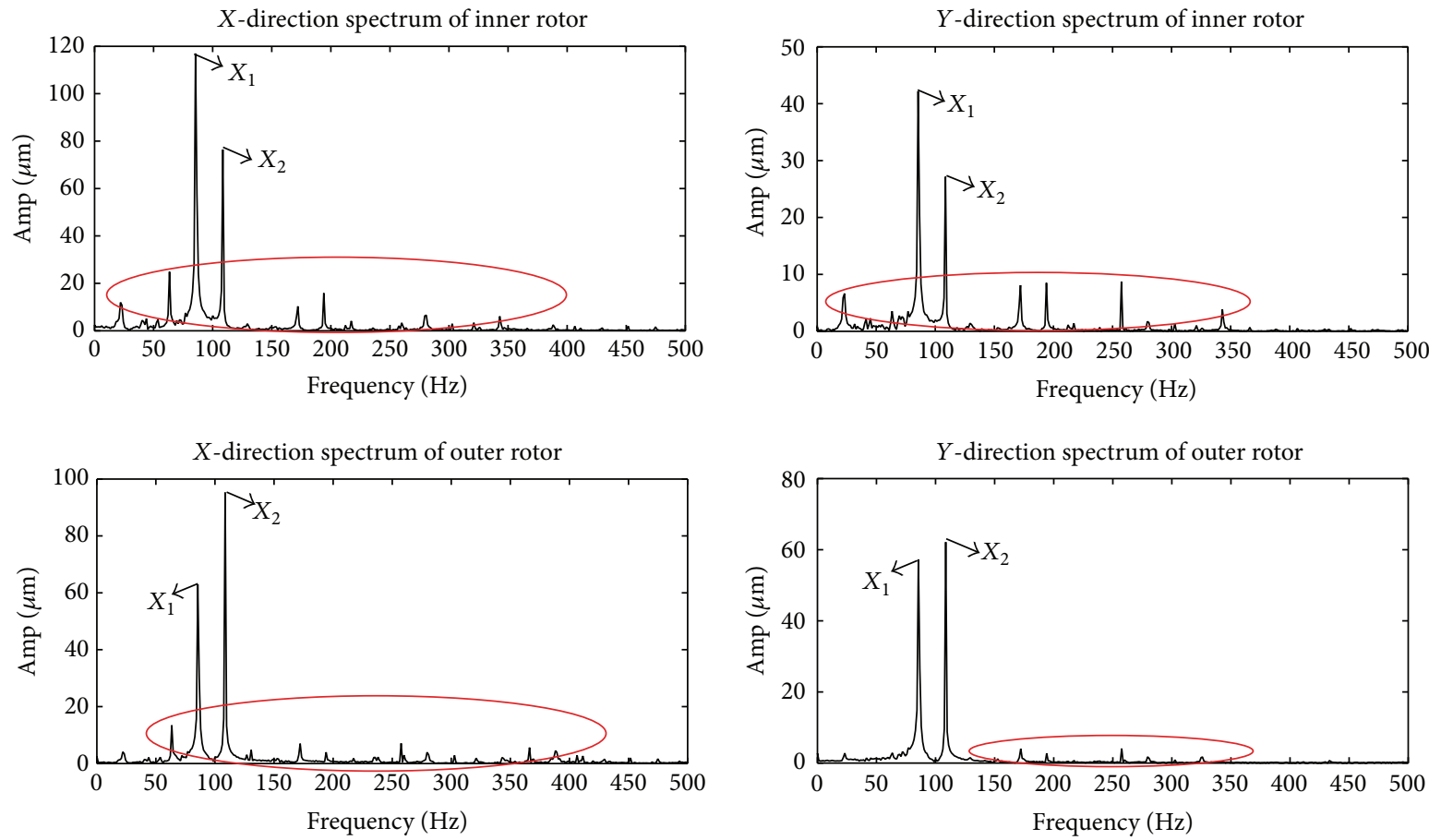

(b) The spectrum with pedestal looseness

FIGURE 3: Vibration spectrum analysis of inner and outer rotor.

values are less than 0 , indicating the sum of the elastic deformation between the pedestal's edges and the foundation along the $Y$-direction in the contact area. The distance between the bolt cap and the foundation is defined as $\delta$ when looseness occurs. The corresponding contact stiffness is expressed as $K_{3 y u}$ when the bolt cap contacts the pedestal's edge. The pedestal moves along $X$-direction, which will cause the bending of bolts and corresponding flexural stiffness is defined as $K_{2 x}$ and $K_{3 x}$, respectively. The stiffness of spring connection between outer ring centre of pedestal and 

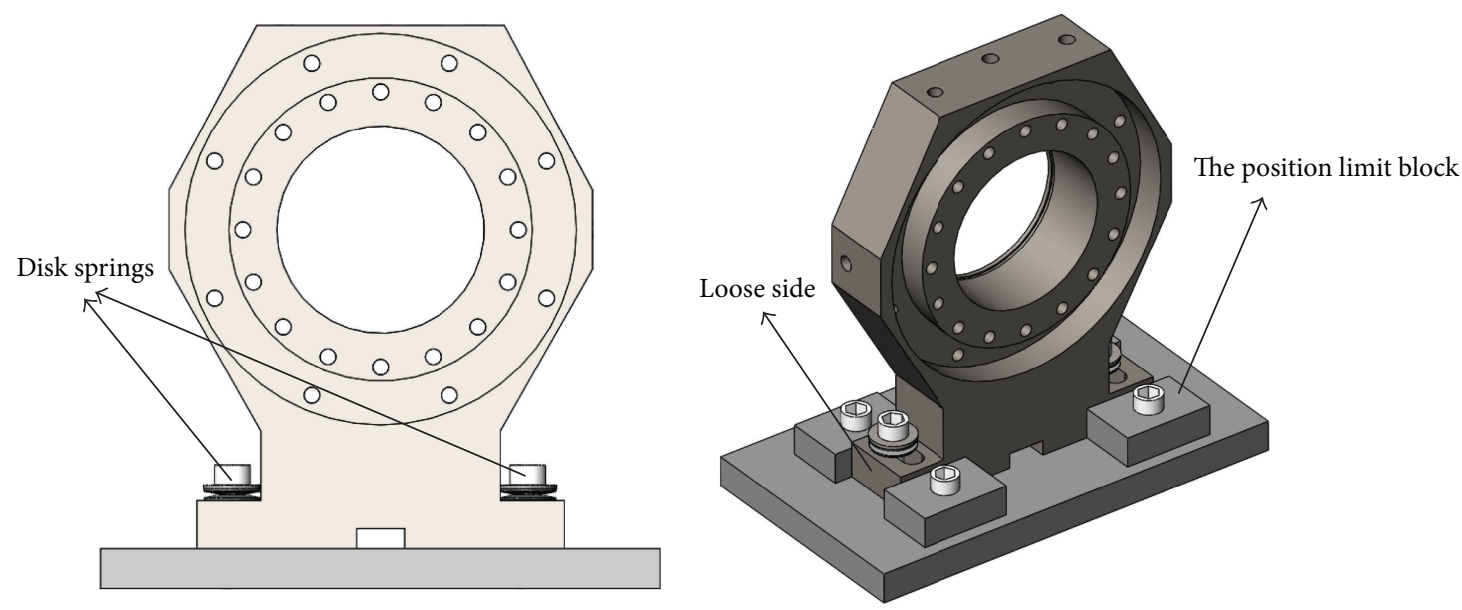

Figure 4: The unilateral loosening states of bearing pedestal.

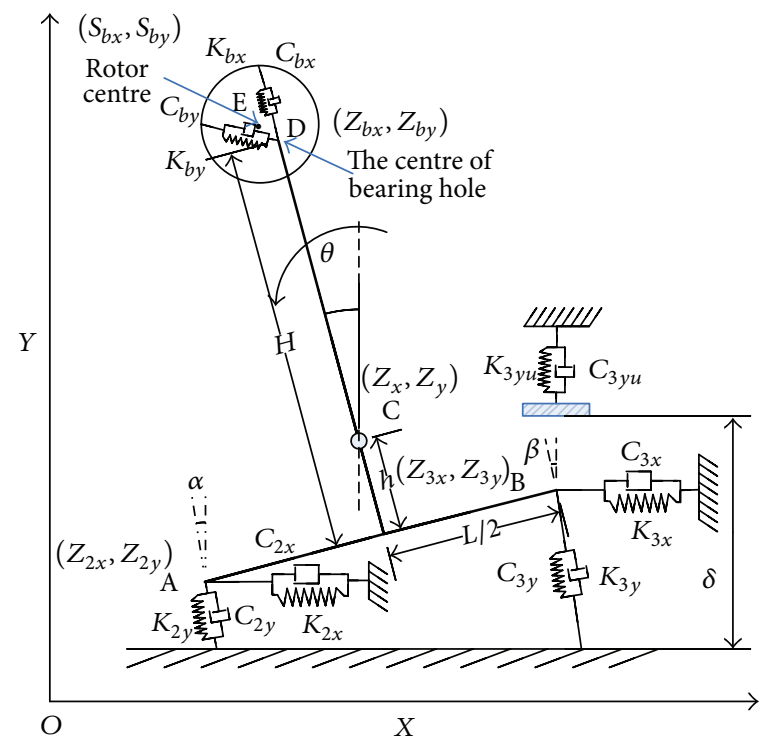

FIgURE 5: Dynamic model of bearing pedestal looseness.

rotor centre is considered supporting stiffness of bearing in $X$ - and $Y$-direction, and the lengths of spring tension or compression are formulated as $d_{1 x}$ and $d_{1 y}$, respectively. For the convenience of drawing the diagram of dynamic model, the spring connection points of rotor centre in $X$ - and $Y$ direction are set at the edge of the rotor. Therefore, the spring stiffness of pedestal's edges in $Y$-direction can be expressed as follows:

$$
\begin{aligned}
& K_{3 y}= \begin{cases}K_{3 y d}, & d_{3 y}<0, \\
K_{3 y m}, & 0<d_{3 y}<\delta, \\
K_{3 y u}, & \delta<d_{3 y},\end{cases} \\
& K_{2 y}= \begin{cases}K_{2 y d}, & d_{2 y}<0, \\
K_{2 y m}, & 0<d_{2 y} .\end{cases}
\end{aligned}
$$

According to the schematic diagram (Figure 4) and geometrical relationship, the expressions of $d_{1 x}, d_{2 x}$, and $d_{3 x}$ are defined as

$$
\begin{aligned}
& d_{1 x}=Z_{x}-S_{x}-(H-h) \theta \\
& d_{1 y}=S_{y}-Z_{y}-(H-h)\left(1-\frac{\theta^{2}}{2}\right) \\
& d_{2 x}=-Z_{x}-h \theta-\frac{L \theta^{2}}{4} \\
& d_{2 y}=Z_{y}+H-h-\frac{L \theta}{2}+\frac{h \theta^{2}}{2} \\
& d_{3 x}=-Z_{x}-h \theta+\frac{L \theta^{2}}{4}, \\
& d_{3 y}=Z_{y}+H-h+\frac{L \theta}{2}+\frac{h \theta^{2}}{2}
\end{aligned}
$$


where $S_{x}$ and $S_{y}$ represent the displacements of $k$ node unit of rotor coupling the pedestal in $X$ - and $Y$-direction.

Based on the dynamics principle and fundamental theory of rigid motion $[18,19]$, the differential equations of motion of the loosened pedestal can be obtained:

$$
\begin{aligned}
m \ddot{Z}_{x}= & -C_{b x} \dot{d}_{1 x}-K_{b x} d_{1 x}+C_{2 x} \dot{d}_{2 x}+K_{2 x} d_{2 x} \\
& +C_{3 x} \dot{d}_{3 x}+K_{3 x} d_{3 x}+C_{2 y} \dot{d}_{2 x}+K_{2 y} d_{2 x} \\
& +C_{3 y} \dot{d}_{3 x}+K_{3 y} d_{3 x}, \\
m \ddot{Z}_{y}= & C_{b y} \dot{d}_{1 y}+K_{b y} d_{1 y}-C_{2 y} \dot{d}_{2 y}-K_{2 y} d_{2 y}-C_{3 y} \dot{d}_{3 y} \\
& -K_{3 y} d_{3 y}-m g, \\
J \ddot{\theta}= & C_{b x} \dot{d}_{1 x} \cdot(H-h)+K_{b x} d_{1 x} \cdot(H-h)+C_{2 x} \dot{d}_{2 x} \\
& \cdot h+K_{2 x} d_{2 x} \cdot h+C_{2 y} \dot{d}_{2 x} \cdot h+K_{2 y} d_{2 x} \cdot h \\
& +C_{3 y} \dot{d}_{2 x} \cdot h+K_{3 y} d_{2 x} \cdot h+C_{3 x} \dot{d}_{3 x} \cdot h \\
& +K_{3 x} d_{3 x} \cdot h+C_{2 y} \dot{d}_{2 y} \cdot \frac{L}{2}+K_{2 y} d_{2 y} \cdot \frac{L}{2} \\
& -C_{3 y} \dot{d}_{3 y} \cdot \frac{L}{2}-K_{3 y} d_{3 y} \cdot \frac{L}{2},
\end{aligned}
$$

where $J$ denotes the inertia moment of $Z$-axis across the gravity centre; $m$ represents the mass of pedestal; $\theta$ is included in the moment expression and can be substituted by $\theta_{i-1}$ when calculated. Arranging formulas (2) (5) the dynamic equations can be rewritten as follows:

$$
\begin{aligned}
M \ddot{Z}_{x}+ & \left(C_{b x}+C_{2 x}+C_{3 x}+C_{2 y}+C_{3 y}\right) \dot{Z}_{x}+\left(K_{b x}\right. \\
+ & \left.K_{2 x}+K_{3 x}+K_{2 y}+K_{3 y}\right) Z_{x}-\left[C_{b x}(H-h)\right. \\
- & \left.\left(C_{2 x}+C_{2 y}\right) h-\left(C_{3 x}+C_{3 y}\right) h\right] \dot{\theta}-\left[K_{b x}(H-h)\right. \\
- & \left.\left(K_{2 x}+K_{2 y}\right) h-\left(K_{3 x}+K_{3 y}\right) h\right] \theta-K_{b x} S_{x} \\
- & C_{b x} \dot{S}_{x}=0 \\
M \ddot{Z}_{y} & +\left(C_{b y}+C_{2 y}+C_{3 y}\right) \dot{Z}_{y}+\left(K_{b y}+K_{2 y}+K_{3 y}\right) Z_{y} \\
- & \frac{L}{2}\left[C_{2 y}-C_{3 y}\right] \dot{\theta}-\frac{L}{2}\left[K_{2 y}-K_{3 y}\right] \theta-C_{b y} \dot{S}_{y} \\
- & K_{b y} S_{y}=-(H-h)\left(K_{b y}+K_{2 y}+K_{3 y}\right)-m g, \\
J \ddot{\theta} & +\left[C_{b x}(H-h)^{2}+\left(C_{2 x}+C_{2 y}\right) h^{2}\right. \\
& \left.+\left(C_{3 x}+C_{3 y}\right) h^{2}\right] \dot{\theta}+\left[K_{b x}(H-h)^{2}\right. \\
& \left.+\left(K_{2 x}+K_{2 y}\right) h^{2}+\left(K_{3 x}+K_{3 y}\right) h^{2}\right] \theta \\
& -\left[C_{b x}(H-h)-\left(C_{2 x}+C_{2 y}\right) h-\left(C_{3 x}+C_{3 y}\right) h\right] \\
& +\dot{Z}_{x}-\left[K_{b x}(H-h)-\left(K_{2 x}+K_{2 y}\right) h\right.
\end{aligned}
$$

$$
\begin{aligned}
& \left.-\left(K_{3 x}+K_{3 y}\right) h\right] Z_{x}-\frac{L}{2}\left[C_{2 y}-C_{3 y}\right] \dot{Z}_{y}-\frac{L}{2}\left[K_{2 y}\right. \\
& \left.-K_{3 y}\right] Z_{y}+C_{b x}(H-h) \dot{S}_{x}+K_{b x}(H-h) S_{x} \\
& =\frac{L}{2}(H-h)\left(K_{2 y}-K_{3 y}\right) .
\end{aligned}
$$

Formulas (6) (8) above can be described in the matrix form as follows:

$$
M_{L} \ddot{Z}+C_{L} \dot{Z}+K_{L} Z=Q_{L},
$$

where $M_{L}$ denotes the mass matrix; $C_{L}$ represents the damping matrix; $K_{L}$ denotes the stiffness matrix; $Q_{L}$ denotes the excitation force vector.

One-dimensional finite element model of dual-rotor is established and the dynamic equation in the matrix form is obtained based on the node map divided in Figure 2. Consider

$$
\left(M_{T}+M_{R}\right) \ddot{Z}+(\Omega G+C) \dot{Z}+\left(K_{B}+K_{b}\right) Z=Q,
$$

where $M_{T}$ and $M_{R}$ represent mass matrix and mass inertia matrix, respectively; $G$ represents gyroscopic matrix; $C$ denotes damping matrix; $K_{B}$ is the bending stiffness matrix of rotor; $K_{b}$ is the support stiffness matrix of rotor; $Q$ denotes the excitation force vector exerted on the nodes of rotor.

Let $K_{Z}=K_{B}+K_{b}, M_{Z}=M_{T}+M_{R}$, and $C_{Z}=\Omega G+C$. It is assumed that the rotor is divided into $n$ nodes and the number of nodes of rotor coupled with the pedestal is $k$. $K_{L}$, $C_{L}, M_{L}$, and $Q_{L}$ and $K_{Z}, C_{Z}, M_{Z}$, and $Q$ are added together. The transient response of the system under pedestal looseness status can be integrally solved based on (6) (8) and dynamic relationship of $k$ th node. Consider

$$
\begin{aligned}
& K_{\text {new }} \\
& =\left[\begin{array}{ccccc}
K_{Z} & & -K_{b x} & & K_{b x}(H-h) \\
& & -K_{b y} & \\
-K_{b x} & & & & \\
& -K_{b y} & & \\
K_{b x}(H-h) & & & K_{L}
\end{array}\right], \\
& C_{\text {new }} \\
& =\left[\begin{array}{cccc}
C_{Z} & & -C_{b x} & C_{b x}(H-h) \\
& & -C_{b y} & \\
-C_{b x} & & & \\
& -C_{b y} & & \\
C_{b x}(H-h) & & & C_{L}
\end{array}\right], \\
& M_{\text {new }}=\left[M_{Z}, M_{L}\right]^{T} \text {, } \\
& Q_{\text {new }}=\left[Q_{1}, \ldots, Q_{4 k-2}+K_{b y}(H-h), \ldots, Q_{4 n}, Q_{L}\right]^{T} \text {. }
\end{aligned}
$$




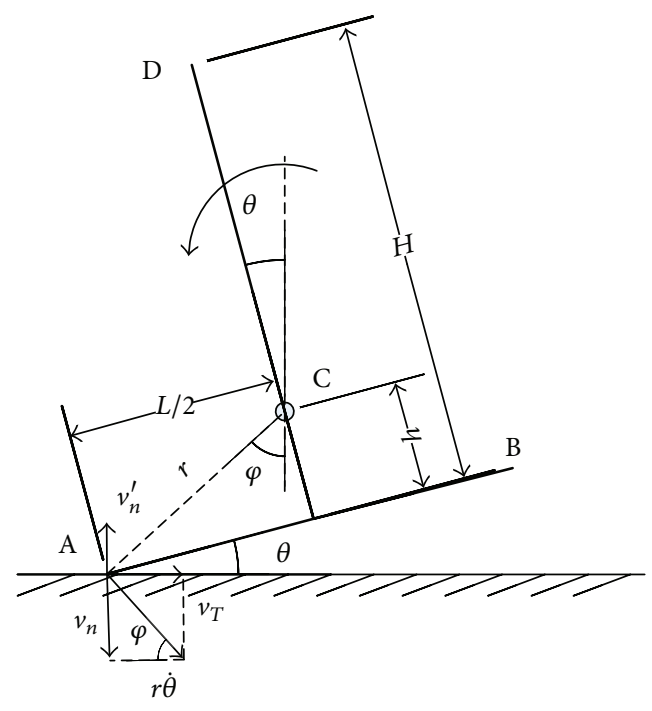

Figure 6: The collision schematic diagram of bearing pedestal and foundation.

Therefore, the dynamic equation integrally solved is obtained:

$$
M_{\text {new }} \ddot{Z}+C_{\text {new }} \dot{Z}+K_{\text {new }} Z=Q_{\text {new }} .
$$

The collision impulse will be exerted on the pedestal when it moves downward to collide with the foundation or moves upward to collide with the bolt cap, which, at the moment of impact, causes the increase of the velocity, but the displacement remains the same in the collision region. In addition, the instantaneous computing instability and even divergence will occur due to the sudden change in the support stiffness of the pedestal. Hence, the collision process should be treated separately. The pedestal and the foundation can be regarded as rigid body, and the local contact deformation is not considered at the moment of collision. The restitution coefficient is defined as follows:

$$
e_{s}=\frac{v_{2}^{\prime}-v_{1}^{\prime}}{v_{1}-v_{2}}
$$

where $v_{1}$ and $v_{2}$ are the speeds of the objects before the collision and $v_{1}>v_{2} ; v_{1}^{\prime}$ and $v_{2}^{\prime}$ are the speeds of the objects after the collision.

In general, $0<\left|e_{s}\right|<1$, and its value is associated with the material, collisional pattern, and so on, so it can be determined by relevant references and experiments. The pedestal is made of rigid material, and the collision that existed between the pedestal and the foundation is considered the frontal collision; let $e_{s}=0.6$. The collision schematic diagram of pedestal and foundation is illustrated in Figure 6.

Here, $r=\left(L^{2} / 4+h^{2}\right)^{1 / 2}$, and $v_{n}$ is rotation linear velocity component of contact point A along the foundation's normal direction before the collision, given by

$$
v_{n}=r \dot{\theta} \sin \varphi .
$$

$v_{n}^{\prime}$ is velocity component of contact point A along the foundation's normal direction after the collision. The foundation is fixed on the ground, so it is suitable to assume that the velocity of the foundation is equal to 0 during the collision.

Based on the definition of restitution coefficient, $v_{n}^{\prime}$ can be expressed as

$$
v_{n}^{\prime}=-e_{s} v_{n} .
$$

In addition, $v_{n}$ and $v_{n}^{\prime}$ can be also decomposed into two parts, namely, the rotation linear velocity component of point $\mathrm{A}$ in the normal direction and the velocity component of mass centre $\mathrm{C}$, presented by

$$
\begin{aligned}
& v_{n}=-r \dot{\theta} \sin \varphi+\dot{Z}_{y}, \\
& v_{n}^{\prime}=-r \dot{\theta}^{\prime} \sin \varphi+\dot{Z}_{y}^{\prime} .
\end{aligned}
$$

Since $\theta \ll 1$, the following formula can be obtained by mean of the geometrical relationship:

$$
r \sin \varphi=\frac{L}{2} \cos \theta-h \sin \theta \approx \frac{L}{2}-h \theta .
$$

According to the momentum theorem and angular momentum conservation theorem, the system can be described by the following equations:

$$
\begin{aligned}
m\left(v_{n}^{\prime}-v_{n}\right) & =I_{n}, \\
J\left(\dot{\theta}^{\prime}-\dot{\theta}\right) & =I_{n}\left(\frac{L}{2} \cos \theta-h \sin \theta\right)=I_{n}\left(\frac{L}{2}-h \theta\right) .
\end{aligned}
$$

Combining the above formulas, the solution can be obtained by

$$
\begin{aligned}
& \dot{Z}_{y}^{\prime} \\
& =\frac{J\left(1+e_{s}\right)(L / 2-h \theta) \dot{\theta}-\left[J e_{s}+m(L / 2-h \theta)^{2}\right] \dot{Z}_{y}}{J-m(L / 2-h \theta)^{2}}, \\
& \dot{\theta}^{\prime} \\
& =\frac{\left[J+m e_{s}(L / 2-h \theta)^{2}\right] \dot{\theta}-m\left(1+e_{s}\right)(L / 2-h \theta) \dot{Z}_{y}}{J-m(L / 2-h \theta)^{2}} .
\end{aligned}
$$

The dynamic equation can be similarly solved when the edge of the bearing pedestal collides with the bolts.

\section{The Simulation Calculation of the Model and Comparison Analysis with Experimental Results}

3.1. The Simulation Calculation of Pedestal Looseness. In this paper Newmark- $\beta$ equation which has low requirement for integral accuracy is utilized for numerical calculation. Based on dynamic equations of dual-rotor system, systematic transient dynamic simulation is accomplished.

Bearings are fastened to bearing pedestal by using Belleville spring and fastening bolt. Bolt coupling stiffness 


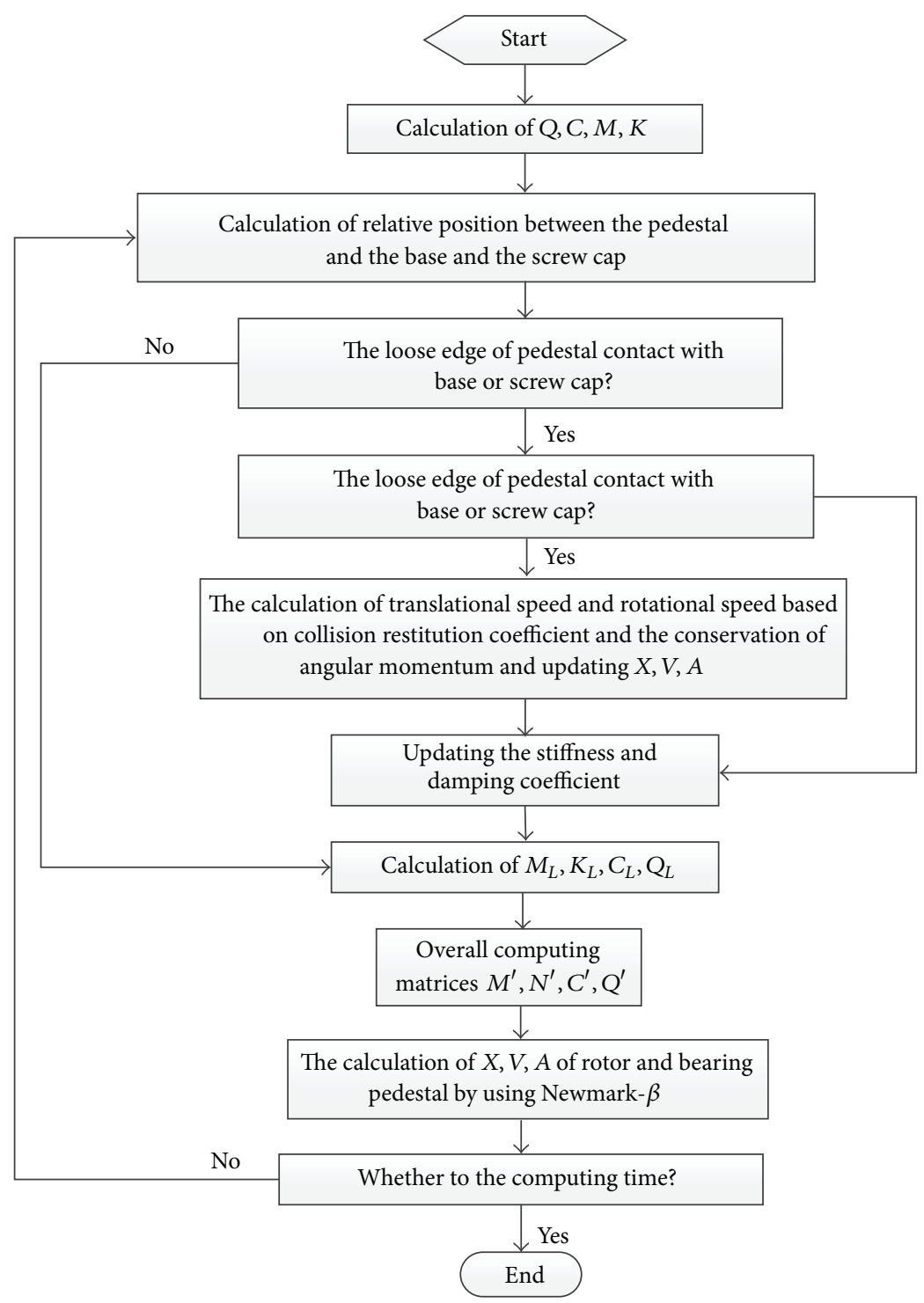

FIGURE 7: Implemented procedure of the proposed dynamic model.

is calculated based on [20]. Under condition of looseness coupling stiffness varies with connection condition of bearing block and bolt due to bearing block's movement. When surface of bolt head is connected to bearing block in its upward movement, the stiffness is about $10^{6}$. Otherwise it is about $10^{2}$. Connection stiffness of bearing block and foundation, bending stiffness of fastening bolt, and bearing support stiffness were calculated according to Hertz theory, beam bending theory, and [21], respectively. Bearing damping value is defined based on [22]. Bolt connection damping value is empirically set. Therefore values for the parameters $K$ and $C$ in the analysis are as follows:

$$
\begin{aligned}
K_{2 y m} & =2.4 e 9 \mathrm{~N} / \mathrm{m}, \\
K_{2 y d} & =3.9 e 10 \mathrm{~N} / \mathrm{m}, \\
K_{3 y m} & =4 e 2 \mathrm{~N} / \mathrm{m},
\end{aligned}
$$

$$
\begin{aligned}
K_{3 y u} & =4 e 6 \mathrm{~N} / \mathrm{m} \\
K_{2 x} & =2 e 10 \mathrm{~N} / \mathrm{m}, \\
K_{3 x} & =2 e 10 \mathrm{~N} / \mathrm{m}, \\
K_{b x} & =4.38 e 8 \mathrm{~N} / \mathrm{m}, \\
K_{b y} & =4.38 e 8 \mathrm{~N} / \mathrm{m}, \\
C_{b x} & =C_{b y}=2 e 4 \mathrm{~N} \cdot \mathrm{s} / \mathrm{m}, \\
C_{2 x} & =C_{2 y}=C_{3 x}=C_{3 y}=2 e 3 \mathrm{~N} \cdot \mathrm{s} / \mathrm{m} .
\end{aligned}
$$

Calculate the following parameters: $\Delta t=0.0001 \mathrm{~s}, \alpha=$ $0.5, \beta=0.5$, and the total time of calculation $T=10 \mathrm{~s}$.

The loose situation is located in 13th node of the inner rotor, as shown in Figure 2. The implemented procedure is demonstrated in Figure 7. 

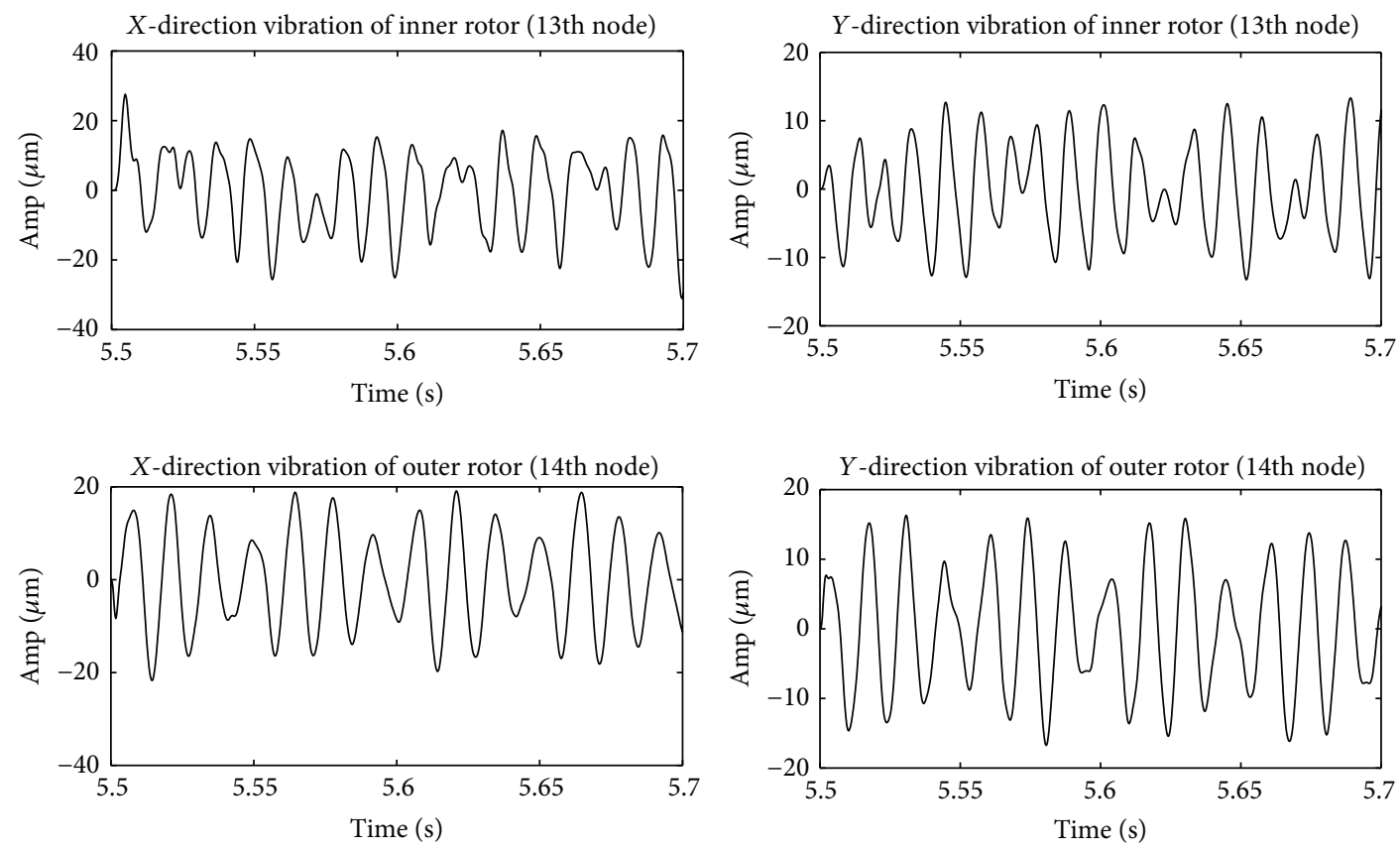

(a) Vibration displacement waveform (loose status)
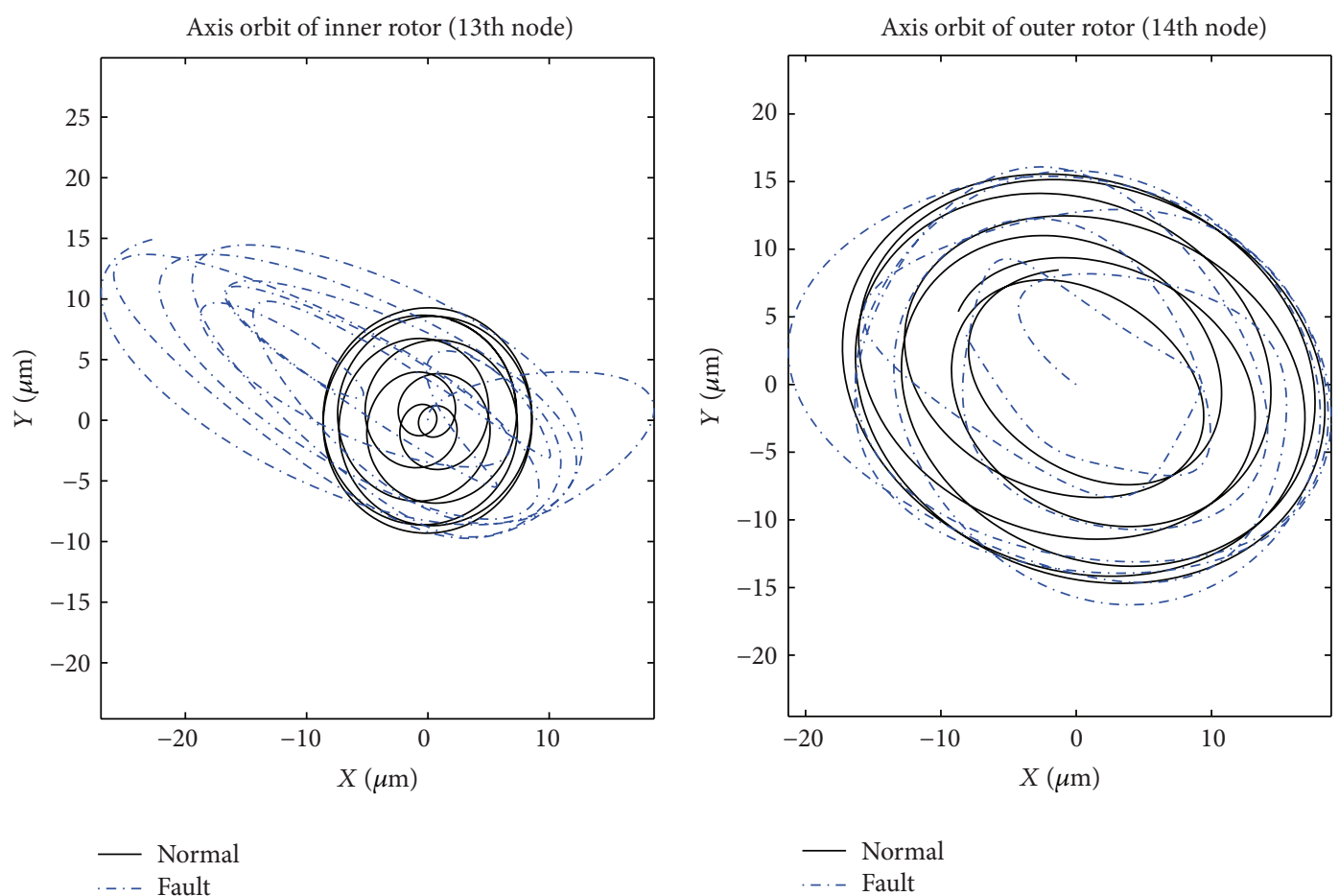

- Normal
-.. Fault

(b) Axis orbits

Figure 8: Dynamic response of inner and outer rotor (loose status).

The rotating speeds of inner rotor and outer rotor are, respectively, set to $4200 \mathrm{rpm}$ and $5400 \mathrm{rpm}$ when the simulation calculation is carried out. The vibration displacement signals of inner rotor and outer rotor are, respectively, collected from 13th node and 14th node to analyse fault characteristics.
(1) The rotating speeds of inner rotor and outer rotor are, respectively, set to $4200 \mathrm{rpm}$ and $5400 \mathrm{rpm}$.

The time waveform of outer rotor and inner rotor with pedestal looseness fault is shown in Figure 8(a); the axis orbits of dual-rotor system with and without looseness fault are illustrated in Figure 8(b); the frequency spectrum 

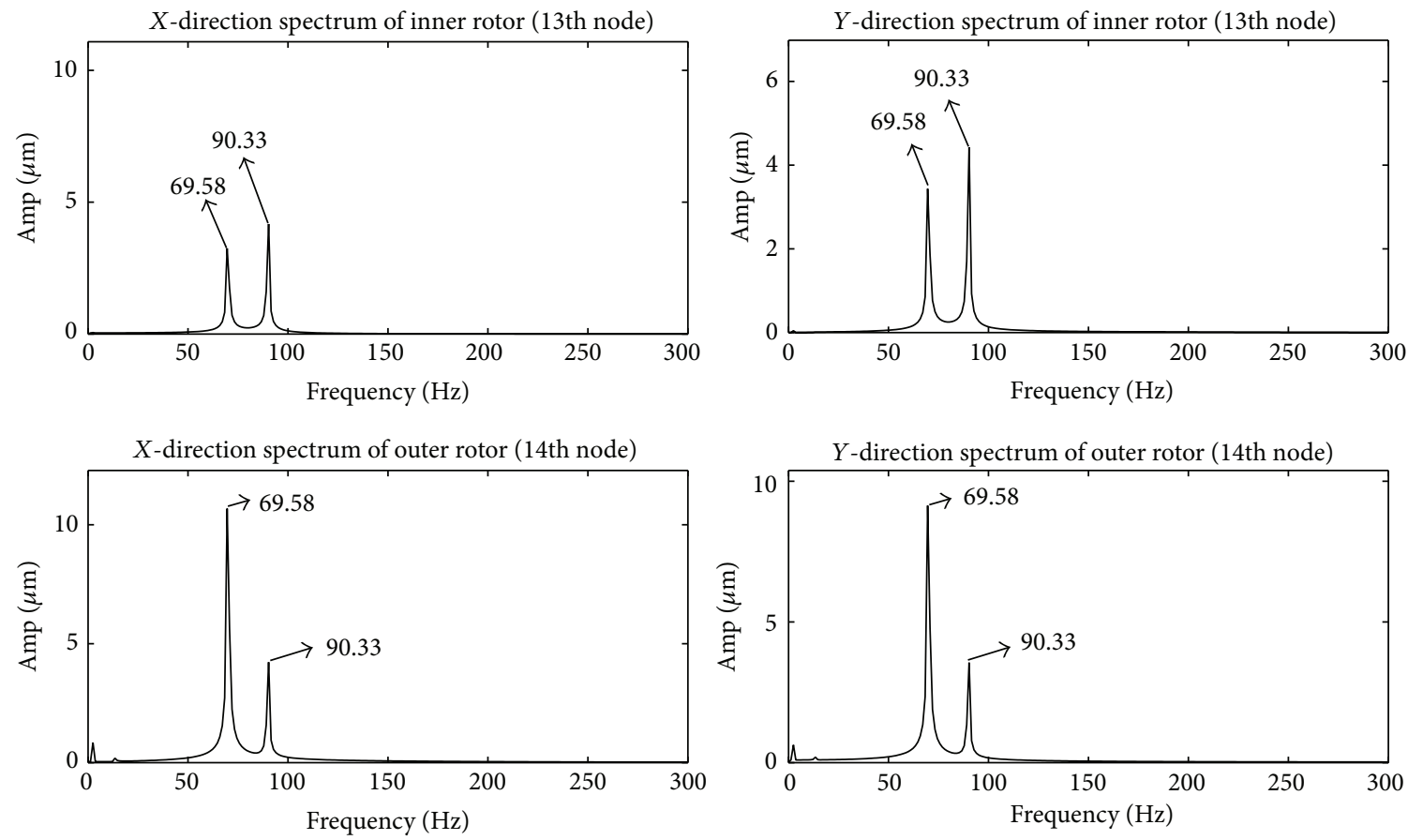

(a) Normal status
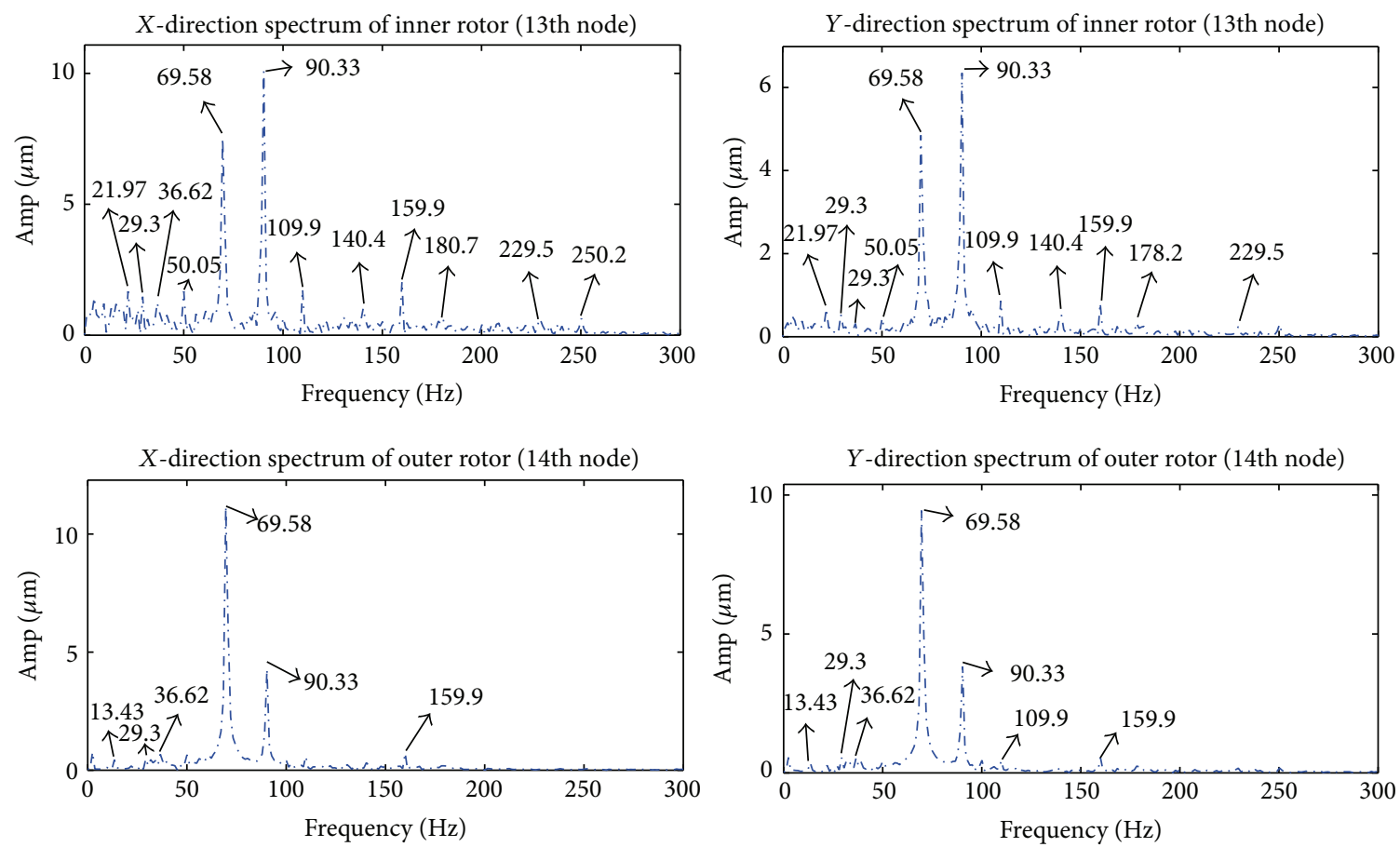

(b) Loose status

FIGURE 9: Vibration spectrum analysis of inner and outer rotor.

plots under normal status and fault status are illustrated in Figure 9.

(2) The rotating speeds of inner rotor and outer rotor are, respectively, set to $5200 \mathrm{rpm}$ and $6500 \mathrm{rpm}$.

The time waveform of outer rotor and inner rotor with pedestal looseness fault is shown in Figure 10(a); the axis orbits of dual-rotor system with and without looseness fault are illustrated in Figure 10(b); the frequency spectrum plots under normal status and fault status are illustrated in Figure 11 .

It can be observed from the vibration displacement waveforms and frequency spectrums of rotors under two 

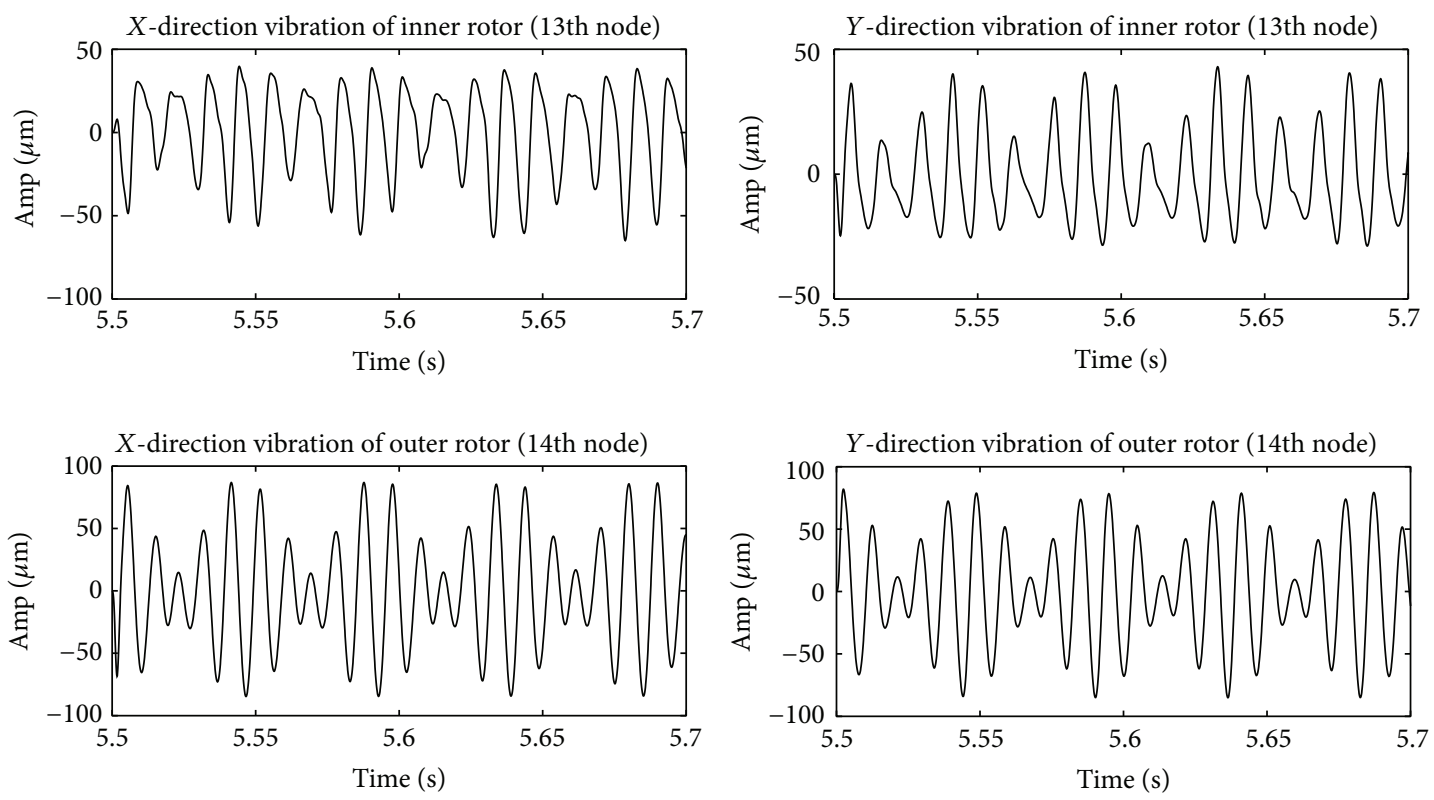

(a) Vibration displacement waveform (loose status)
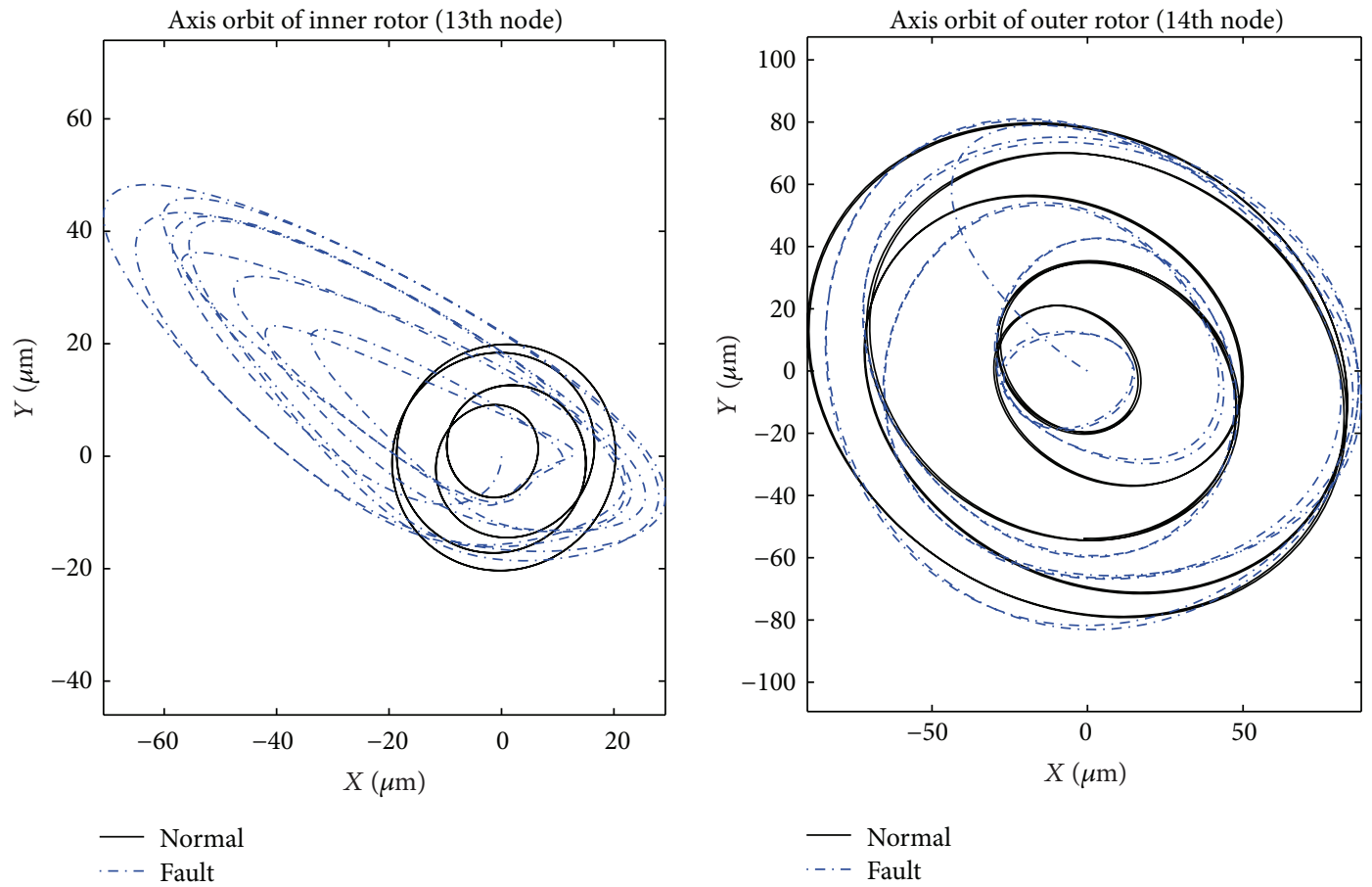

(b) Axis orbits

FIGURE 10: Dynamic response of inner and outer rotor (loose status).

kinds of different speed combination that the vibration displacement of the node located in the looseness position of inner rotor presents the asymmetry in vertical direction and waveform cutting phenomenon when pedestal looseness fault occurs. At the fundamental frequency, the amplitudes of inner rotor's loose node in $X$-direction and $Y$-direction are greater than the amplitudes under normal condition; the amplitudes of node located in the bearing that supported the outer rotor basically remain the same. Besides, multiple harmonic components $2 X_{1}\left(1 X_{1}\right.$ represents the fundamental of inner rotor) and $2 X_{2}\left(1 X_{2}\right.$ represents the fundamental of outer rotor) and sometimes difference frequency and sum frequency components of inner rotor and outer rotor are present; the low frequency components such as $(1 / 2) X_{1}$, 

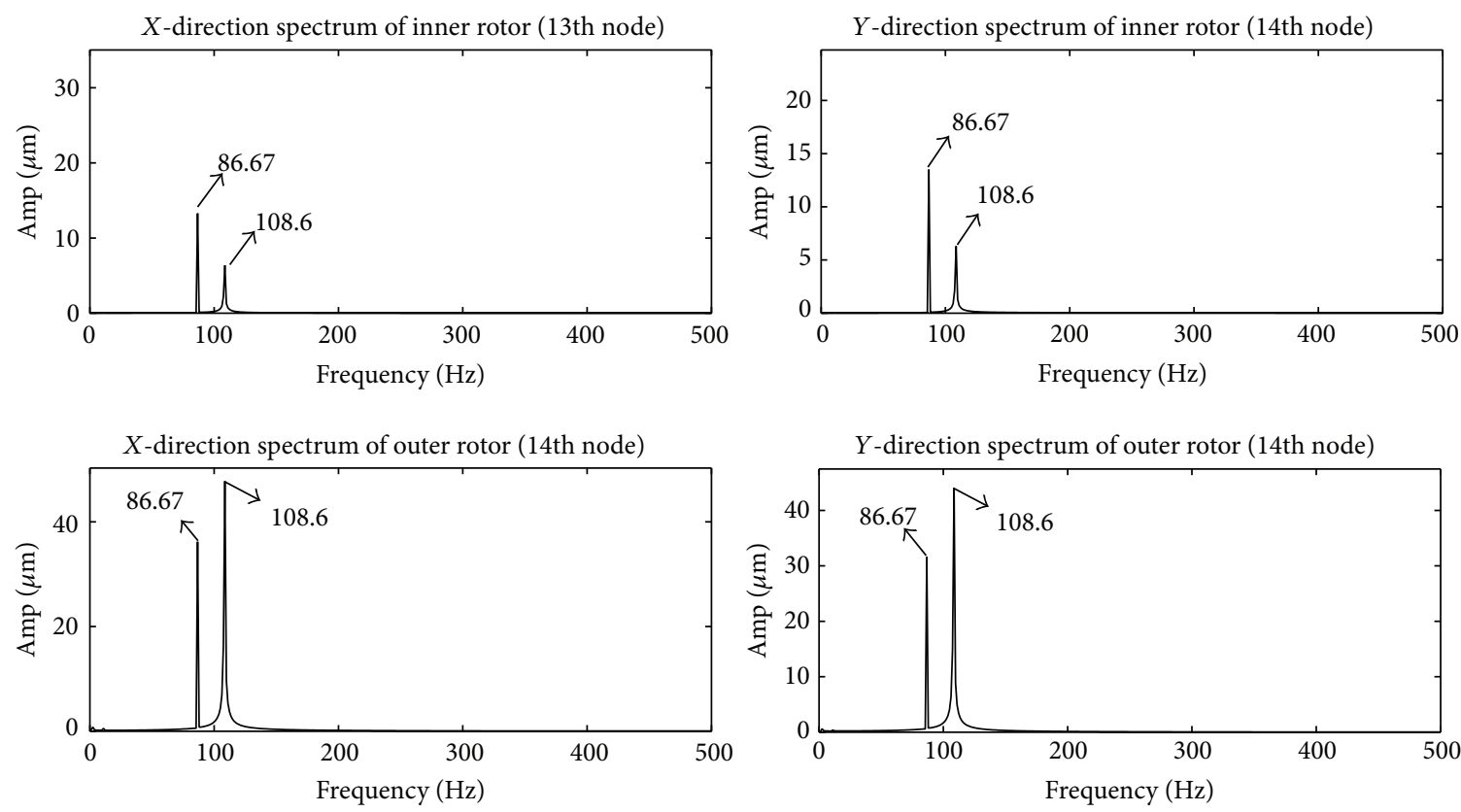

(a) Normal status
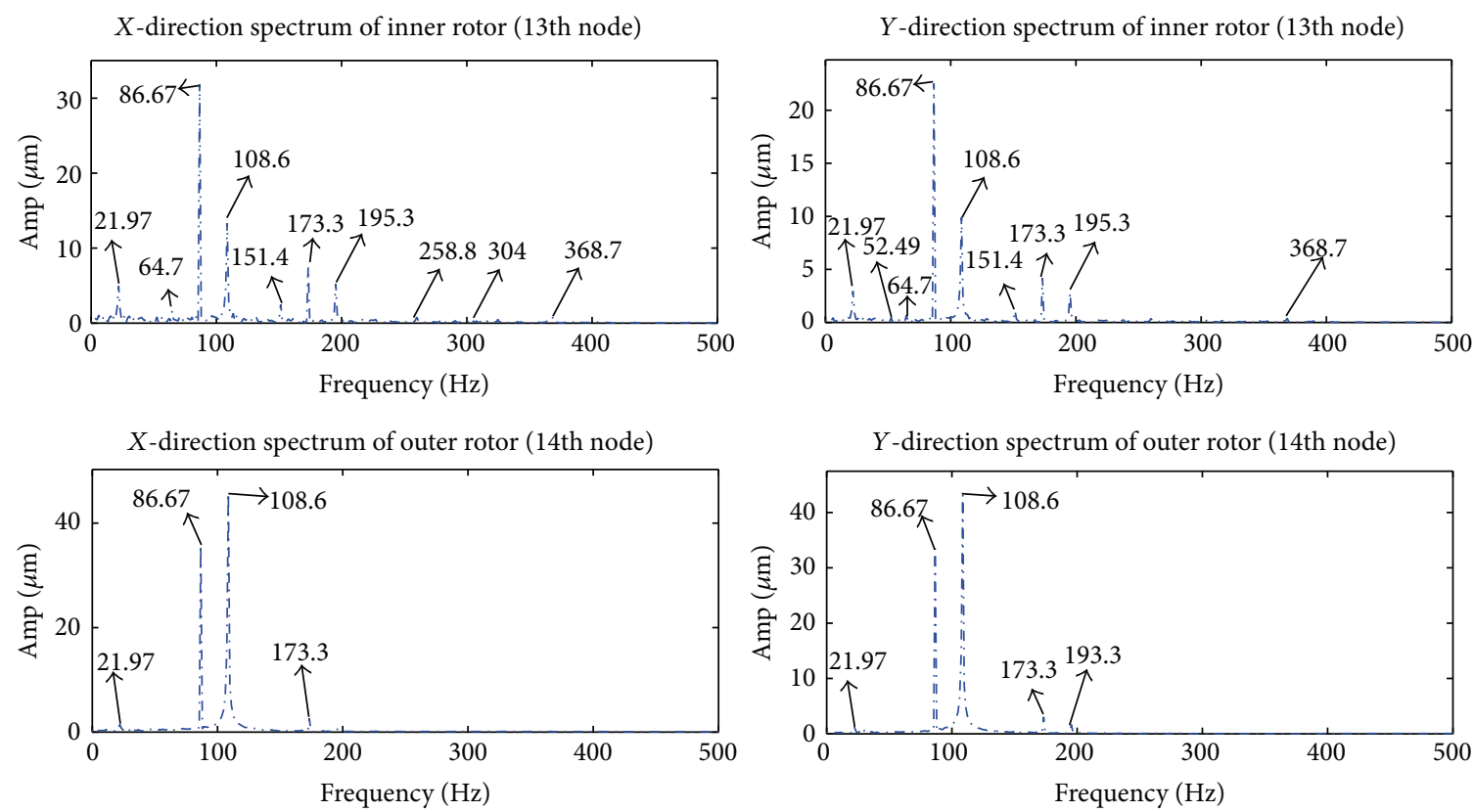

(b) Loose status

FIGURE 11: Vibration spectrum analysis of inner and outer rotor.

$(1 / 2) X_{2},(1 / 3) X_{1}$, and $(1 / 3) X_{2}$ increase in the spectrums of inner and outer rotor. The simulation results are in accordance with the conclusions of the literature [23].

It may be further noticed from the axis orbits that the axis orbit of inner rotor presents thin strips when the bearing pedestal looseness fault of inner rotor occurs, but the axis orbit of outer rotor has almost no change.
3.2. Experimental Verification. In order to verify the efficiency of the theoretical model, the unilateral looseness fault of inner rotor's bearing pedestal is simulated in the dual-rotor system test rig. The schematic of test rig is shown in Figure 12.

The loose position of pedestal of inner rotor is shown in Figure 12, which is corresponding to the 13th node (the roller bearing pedestal of inner rotor) shown in Figure 2. The 


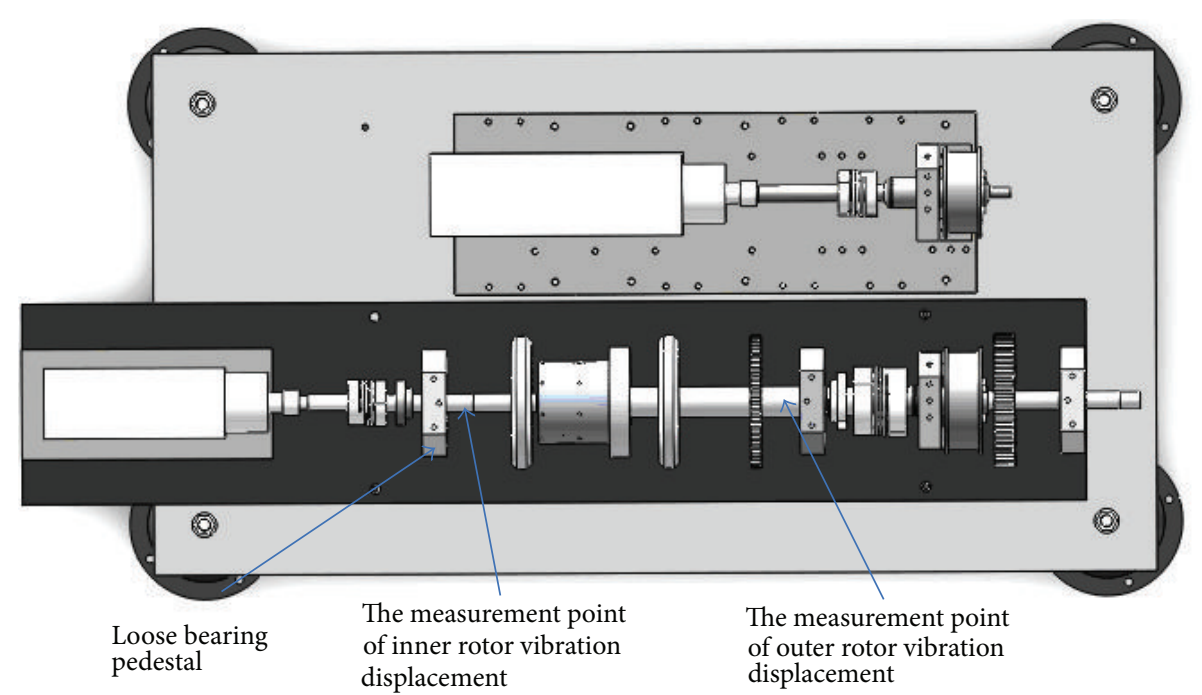

FIGURE 12: Schematic diagram of dual-rotor system loose experiment.

eddy current displacement sensor utilized to collect vibration displacement of inner rotor is installed in the vicinity of the 4th bearing, and vibration displacements of outer rotor are measured by the other displacement sensor, as illustrated in Figure 12. Two cases of different speed combination are demonstrated for experiment, in order to correspond to the model simulation: the rotating speed of inner rotor $4200 \mathrm{rpm}$ and the rotating speed of outer rotor $5400 \mathrm{rpm}$, respectively, and the rotating speed of inner rotor $5200 \mathrm{rpm}$ and the rotating speed of outer rotor $6500 \mathrm{rpm}$, respectively.

(1) The rotating speed of inner rotor is $4200 \mathrm{rpm}$, and the rotating speed of outer rotor is $5400 \mathrm{rpm}$.

The time waveforms, axis orbits, and spectrums of inner and outer rotor are illustrated in Figures 13 and 14.

(2) The rotating speed of inner rotor is $5200 \mathrm{rpm}$, and the rotating speed of outer rotor is $6500 \mathrm{rpm}$.

The time waveforms, axis orbits, and spectrums of inner and outer rotor are illustrated in Figures 15 and 16.

It is observed from the experimental results that vibration displacement waveforms of inner and outer rotor are accompanied with the "waveform cutting," and the phenomenon is more serious for inner rotor. The spectrums of inner rotor vibration displacement exhibit complicated frequency components. Comparing the spectrums of a rotor system with pedestal looseness in the literature $[3,4]$, the spectrum not only contains the fundamental frequencies of inner and outer rotor, $1 / 2$ fractional harmonic components, but also contains sum frequency and difference frequency of two rotors' fundamental frequencies, and superharmonic frequencies (such as $2 X, 3 X$, and $4 X$ ) are presented. In addition, the spectrums also contain combined frequencies of fundamental frequencies of inner and outer rotor. The spectrum components of outer rotor vibration displacement waveforms are relatively simple, and the amplitude is smaller.

It may be noted that the axis orbit of outer rotor has no obvious change comparing to the axis orbit under normal status. However, the axis orbit of inner rotor is drawn into similar-elliptic shape.

\section{Conclusions}

In the study, the dynamic model of dual-rotor system has been proposed to describe the mechanics of pedestal looseness between the pedestal and the foundation and bolt cap. The loose bearing pedestal is regarded as the rigid body with three degrees of freedom. The dynamics equations are numerically integrated for cases with and without pedestal looseness, and the vibration displacement signals are obtained for the two cases. The vibration signals collected from the dynamic analysis and experiments are treated with the Fourier transformation. Comparing with the actual experimental results, the dynamic model is shown to be accurate and effective. Typical conclusions are summarized as follows:

(1) A new dynamic model of dual-rotor system with pedestal looseness considering the effects of the collision is presented. The pedestal looseness model has satisfactory accuracy under some conditions by comparing simulation with experiment, which provides theoretical support for extracting pedestal looseness fault characteristics.

(2) The vibration displacement waveforms of rotors exhibit the "waveform cutting" characteristics. The spectrums of vibration displacement of inner and outer rotor not only contain the sum frequency and difference frequency of two rotors' fundamental frequencies but also contain some superharmonic components (such as $2 X$ and $3 X$ ), and, especially for the loose rotor, the multiple harmonic components are more prominent. Besides the low frequency spectrum is abundant; sometimes fractional harmonic 

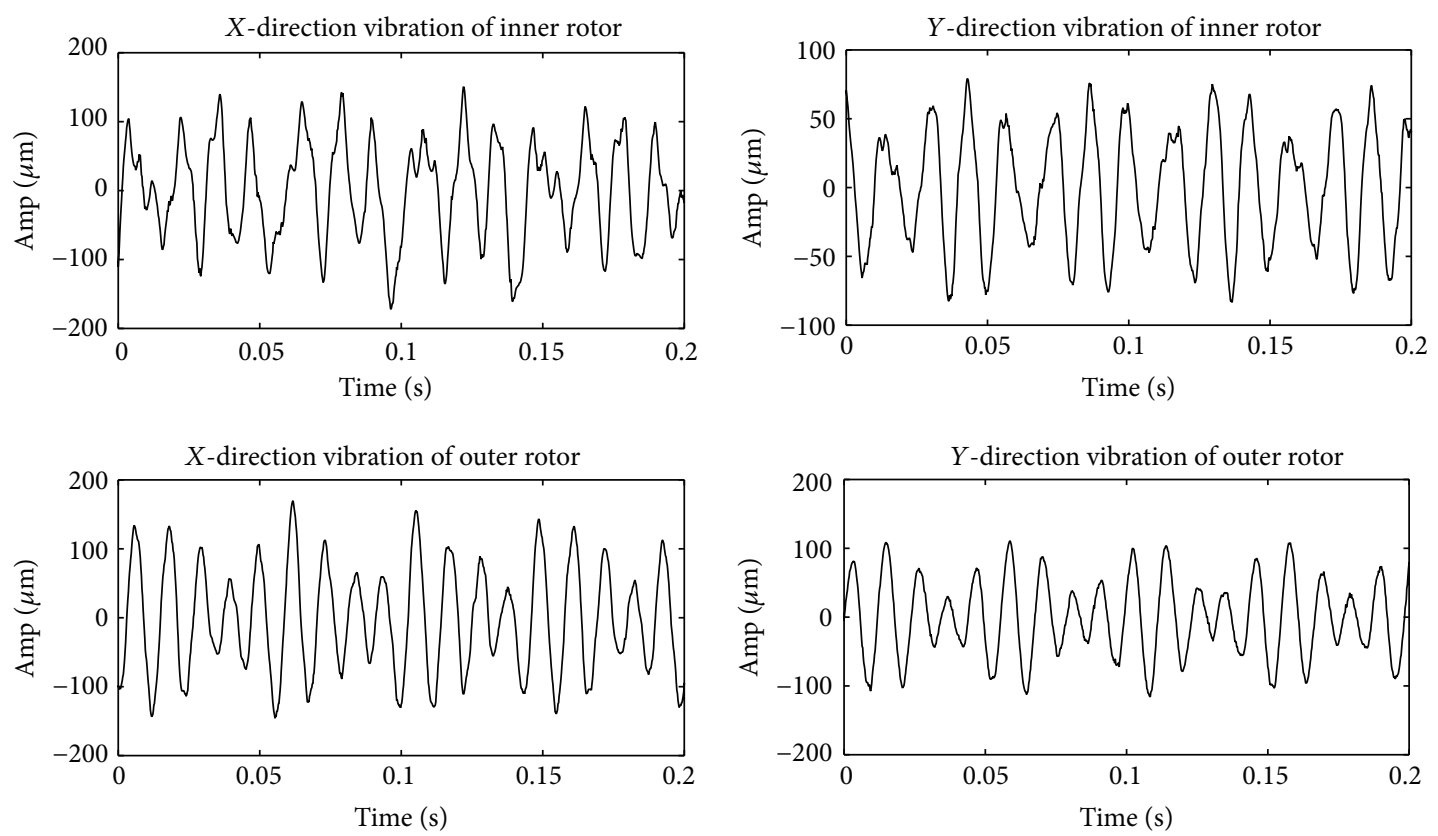

(a) Vibration displacement waveform (loose status)
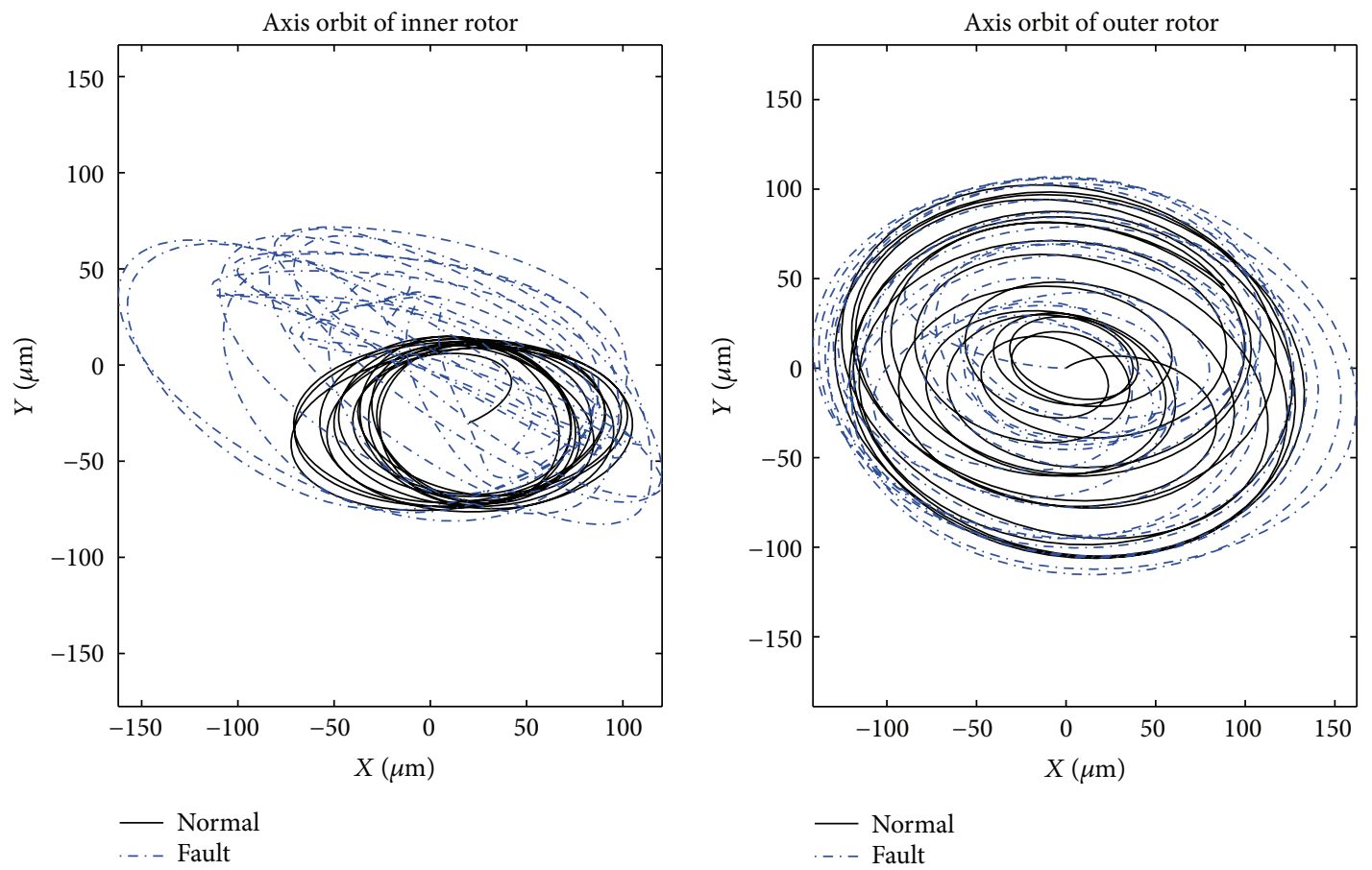

(b) Axis orbits

FIGURE 13: Dynamic response of inner and outer rotor (loose status). 

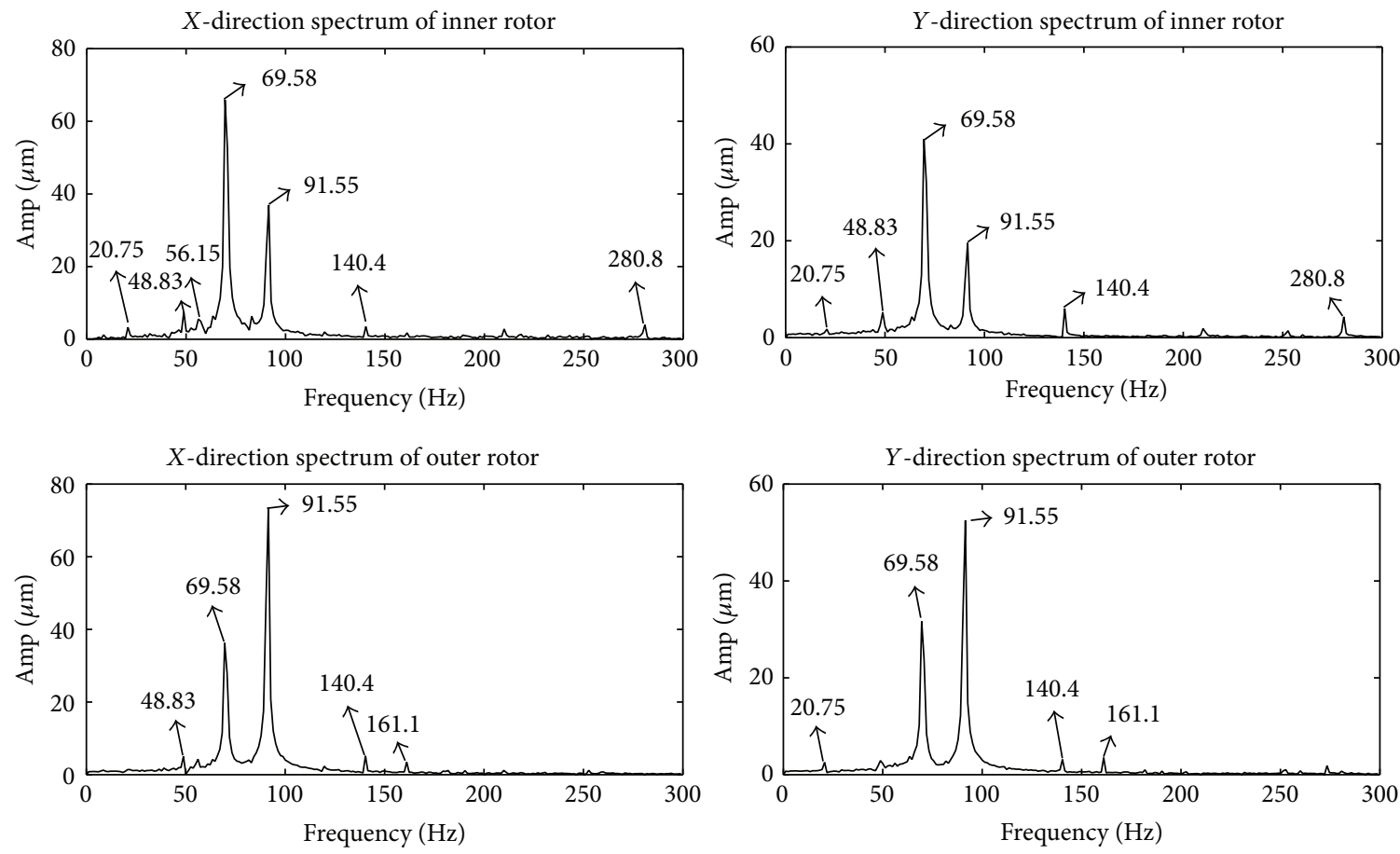

(a) Normal status
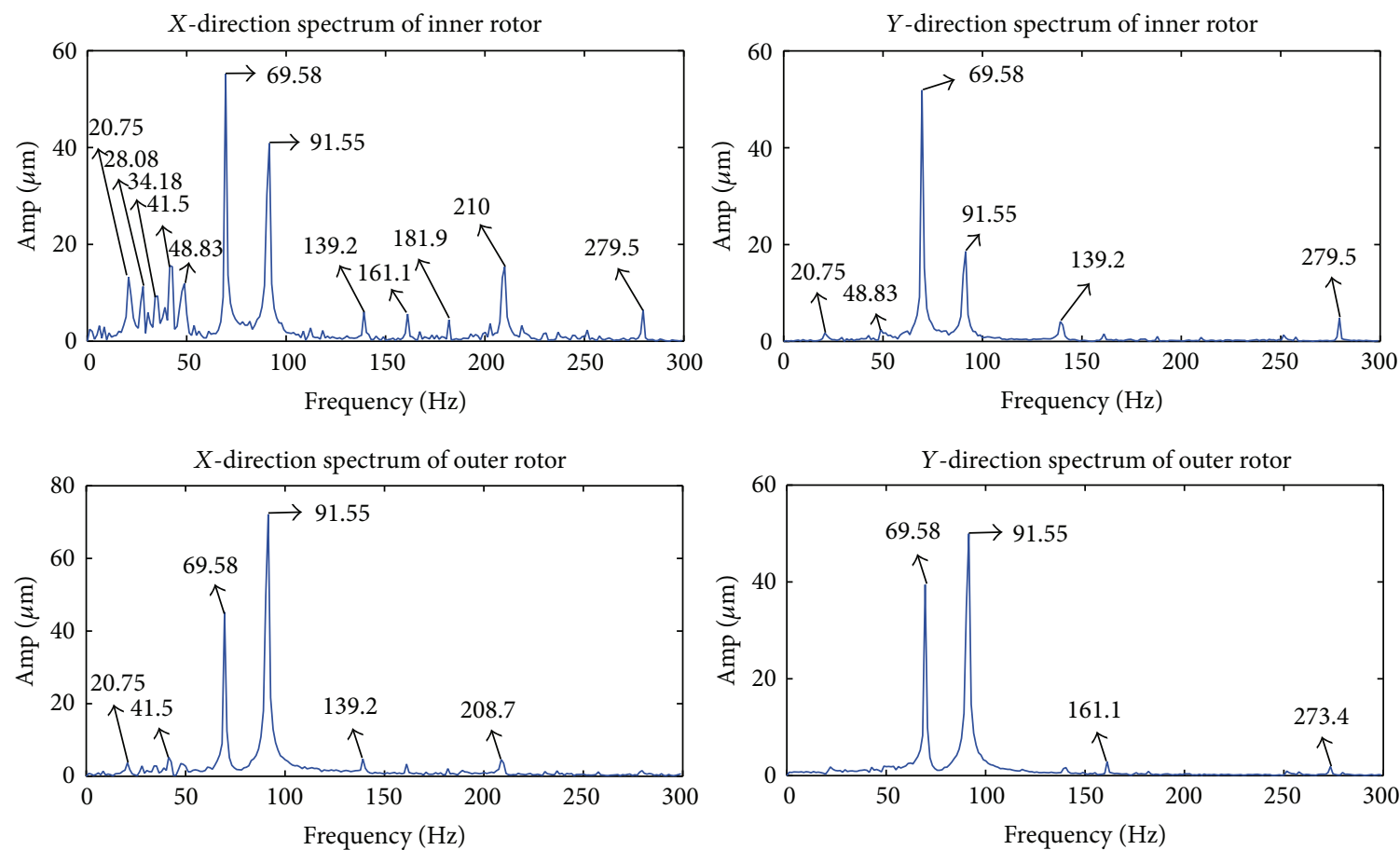

(b) Loose status

FIGURE 14: Vibration spectrum analysis of inner and outer rotor. 

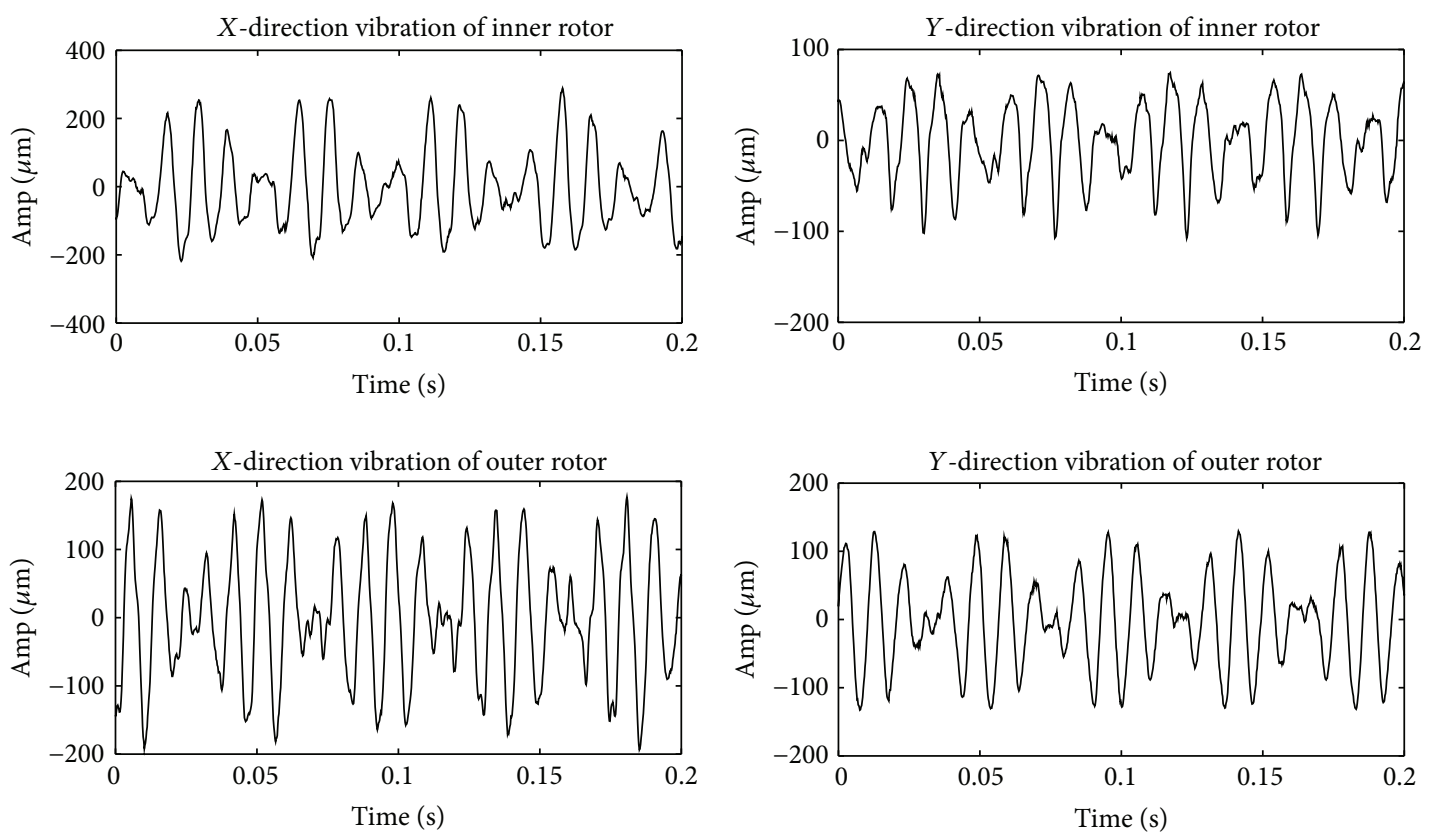

(a) Vibration displacement waveform (loose status)
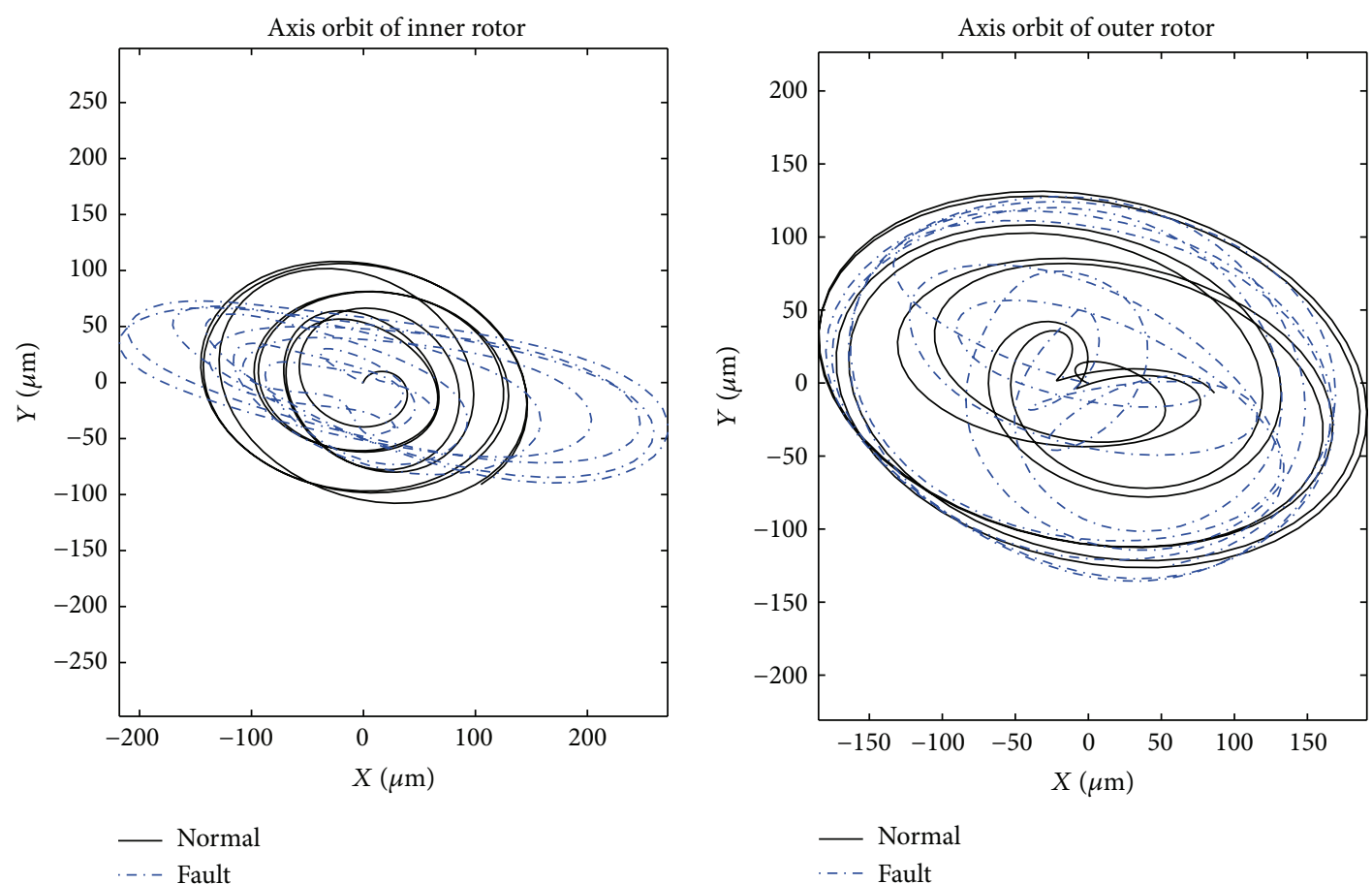

- Normal

-..- Fault

(b) Axis orbits

FIGURE 15: Dynamic response of inner and outer rotor (loose status). 

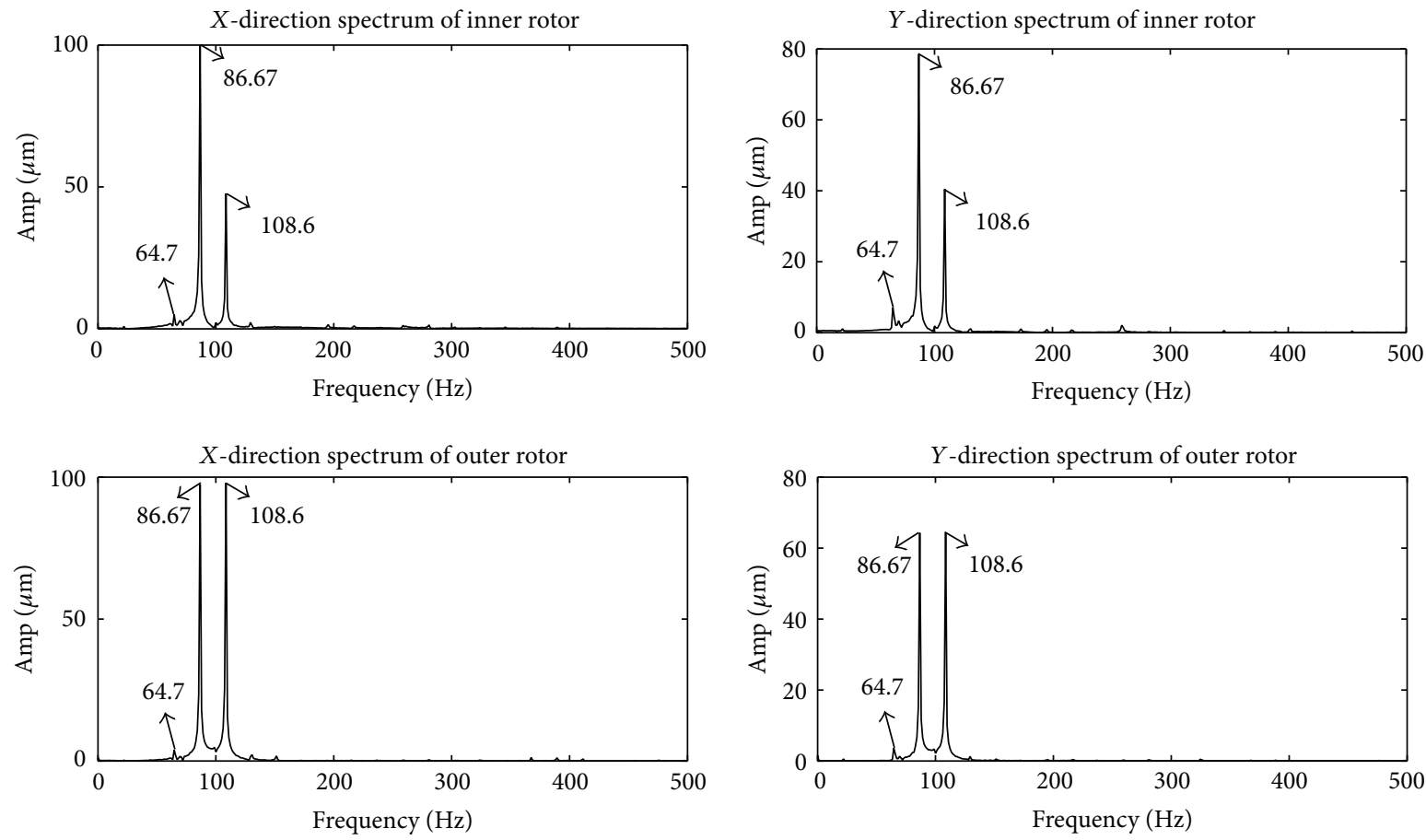

(a) Normal status
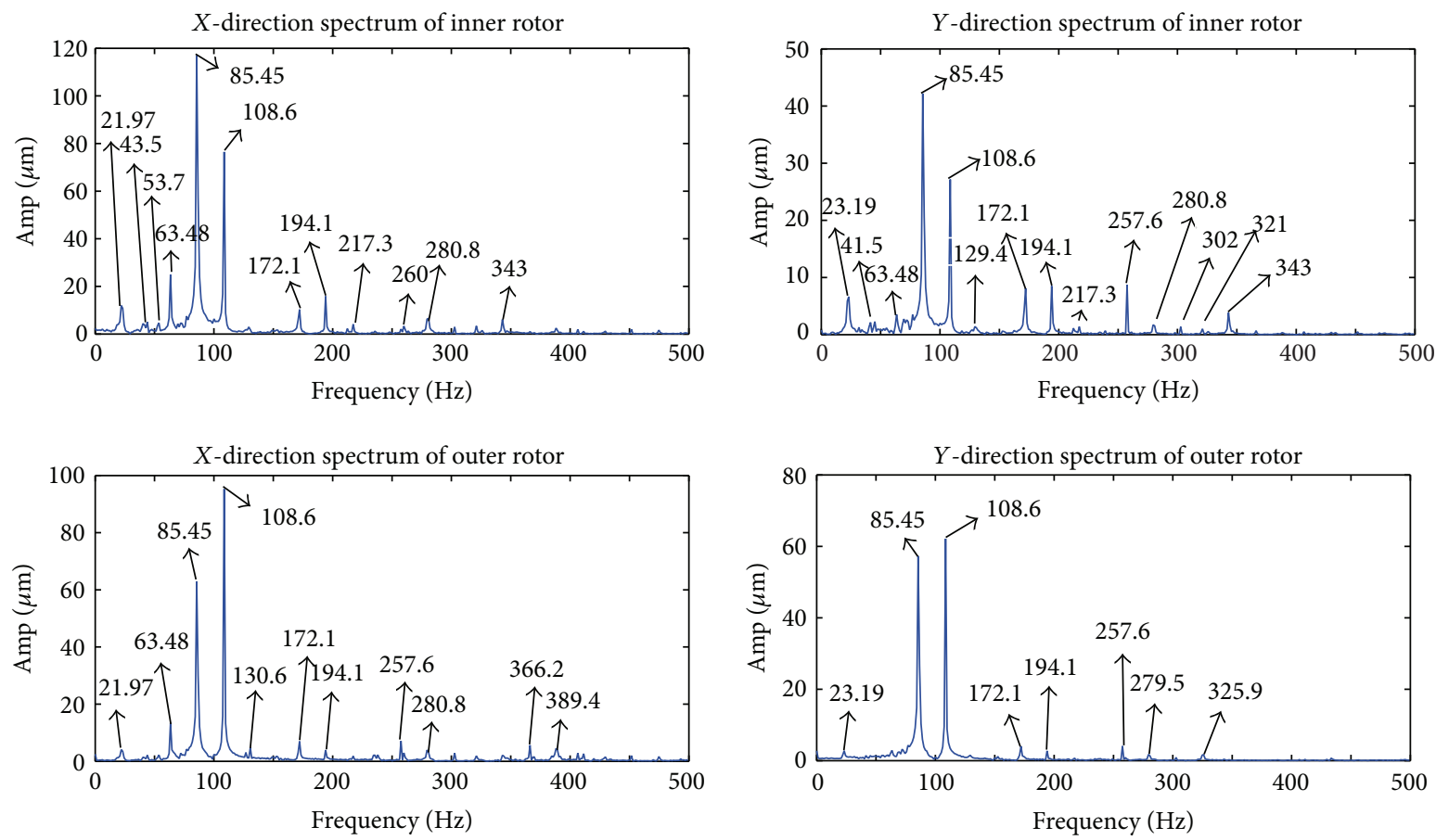

(b) Loose status

FIGURE 16: Vibration spectrum analysis of inner and outer rotor. 
components are present. Some combined frequency components of fundamental frequencies in high frequency are illustrated.

\section{Competing Interests}

The authors declare that there are no competing interests in regard to publishing of the paper.

\section{Acknowledgments}

The project is supported by the National Natural Science Foundations of China (no. 11572167).

\section{References}

[1] P. Goldman and A. Muszynska, "Analytical and experimental simulation of loose pedestal dynamic effects on a rotating machine vibrational response," Rotating Machinery and Vehicle Dynamics, American Society of Mechanical Engineers, vol. 35, pp. 11-17, 1991.

[2] A. Muszynska and P. Goldman, "Chaotic responses of unbalanced rotor/bearing/stator systems with looseness or rubs," Chaos, Solitons and Fractals, vol. 5, no. 9, pp. 1683-1704, 1995.

[3] F. Chu and Y. Tang, "Stability and non-linear responses of a rotor-bearing system with pedestal looseness," Journal of Sound and Vibration, vol. 241, no. 5, pp. 879-893, 2001.

[4] H. Ma, X. Zhao, Y. Teng, and B. Wen, "Analysis of dynamic characteristics for a rotor system with pedestal looseness," Shock and Vibration, vol. 18, no. 1-2, pp. 13-27, 2011.

[5] W. Lu and F. Chu, "Experimental investigation of pedestal looseness in a rotor-bearing system," Key Engineering Materials, vol. 413-414, pp. 599-605, 2009.

[6] Z. Ji and J. W. Zu, "Method of multiple scales for vibration analysis of rotor-shaft systems with non-linear bearing pedestal model," Journal of Sound and Vibration, vol. 218, no. 2, pp. 293305, 1998.

[7] H. Ma, Z. H. Ren, H. L. Yao, F. Wen, and B. C. Wen, "Numerical simulation and experimental research on pedestal looseness of a rotor system," in Proceedings of the ASME International Design Engineering Technical Conferences and Computers and Information in Engineering Conference, pp. 895-901, September 2007.

[8] K. Lu, Y. Jin, Y. Chen, Q. Cao, and Z. Zhang, "Stability analysis of reduced rotor pedestal looseness fault model," Nonlinear Dynamics, vol. 82, no. 4, pp. 1611-1622, 2015.

[9] Z. Qin, Q. Han, and F. Chu, "Bolt loosening at rotating joint interface and its influence on rotor dynamics," Engineering Failure Analysis, vol. 59, pp. 456-466, 2016.

[10] T. Y. Wu, Y. L. Chung, and C. H. Liu, "Looseness diagnosis of rotating machinery via vibration analysis through HilbertHuang transform approach," Journal of Vibration \& Acoustics, vol. 132, no. 3, pp. 1015-1016, 2010.

[11] S.-M. Lee and Y.-S. Choi, "Fault diagnosis of partial rub and looseness in rotating machinery using Hilbert-Huang transform," Journal of Mechanical Science and Technology, vol. 22, no. 11, pp. 2151-2162, 2008.

[12] H. L. Meng and M. S. Leong, "Diagnosis for loose blades in gas turbines using wavelet analysis," Journal of Engineering for Gas Turbines \& Power, vol. 127, no. 2, pp. 289-297, 2003.
[13] Y. He, Z. Chen, D. Guo, and F. Chu, "A genetic algorithm based inverse problem approach for pedestal looseness identification in rotor-bearing systems," Key Engineering Materials, vol. 245246, pp. 115-122, 2003.

[14] H. Ma, J. Huang, S. Zhang, and H. Niu, "Nonlinear vibration characteristics of a rotor system with pedestal looseness fault under different loading conditions," Journal of Vibroengineering, vol. 15, no. 1, pp. 406-418, 2013.

[15] H. F. Wang and G. Chen, "Certain type turbofan engine whole vibration model with support looseness fault and casing response characteristics," Shock and Vibration, vol. 2014, Article ID 683469, 23 pages, 2014.

[16] M. Behzad and M. Asayesh, "Numerical and experimental investigation of the vibration of rotors with loose discs," Proceedings of the Institution of Mechanical Engineers, Part C: Journal of Mechanical Engineering Science, vol. 224, no. 1, pp. 85-94, 2010.

[17] H. F. Wang, G. Chen, and P. P. Song, "Asynchronous vibration response characteristics of aero-engine with support looseness fault," Journal of Computational \& Nonlinear Dynamics, vol. 11, no. 3, Article ID 031013, 2016.

[18] S. Hong, Advanced Dynamics, Tongji University Press, Shanghai, China, 1996.

[19] S. Yuan and Z. Lv, Multi Rigid Body System Dynamics, Beijing Institute of Technology Press, Beijing, China, 1992.

[20] Y. Wu, Vibration Analysis of Aero-Engine Considering Connecting Stiffness and Damping, Nanjing University of Aeronautics and Astronautics, Nanjing, China, 2010.

[21] Z. Chen, "Stiffness computaion of rolling bearing and its support," Coal Mine Machinery, vol. 3, no. 27, pp. 387-388, 2006.

[22] H. Wu, J. Wang, and A. N. Qi, "Calculating method for damping of cylindrical roller bearings," Bearing, vol. 9, pp. 1-5, 2008.

[23] D. Yang and Z. Liu, "Diagnosis of vibration fault generated by the loose of elasticity backup bearing on aeroengine's rotor," Journal of Test and Measurement Technology, vol. 26, no. 4, pp. 7-10, 2007. 


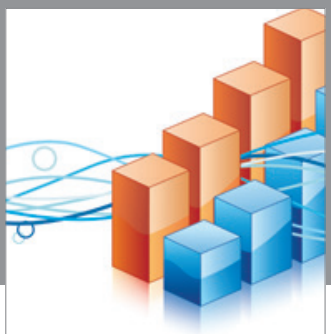

Advances in

Operations Research

vatem alat4

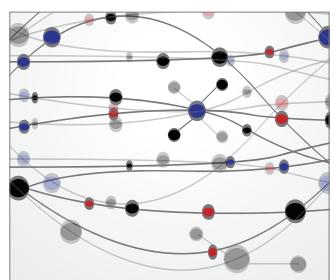

\section{The Scientific} World Journal
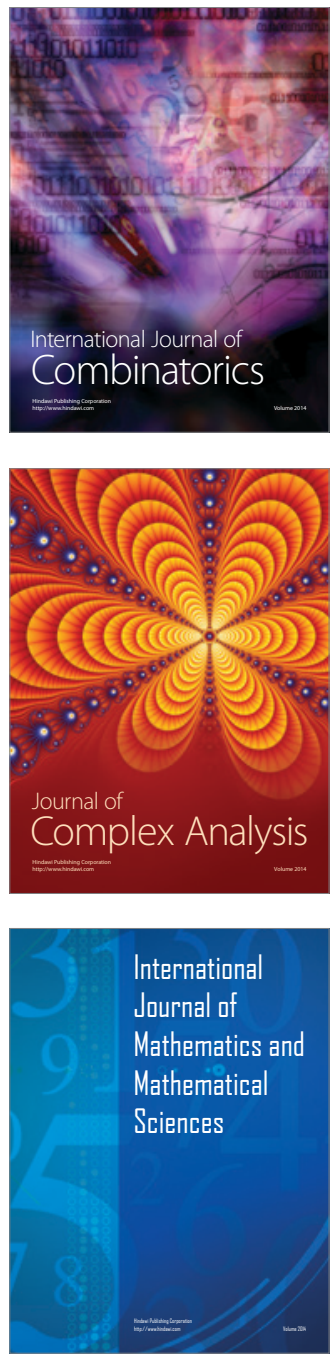
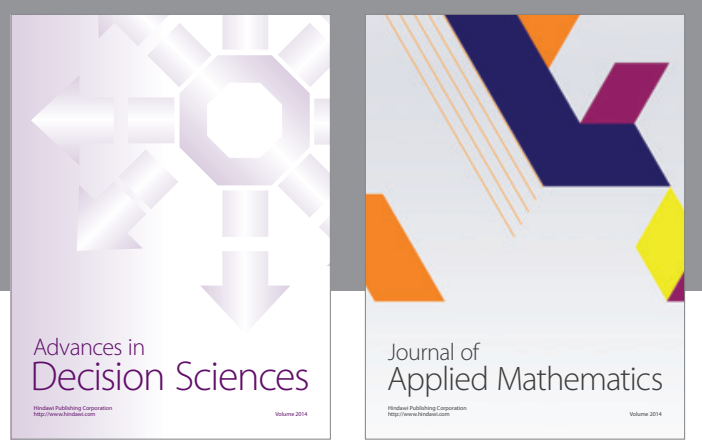

Algebra

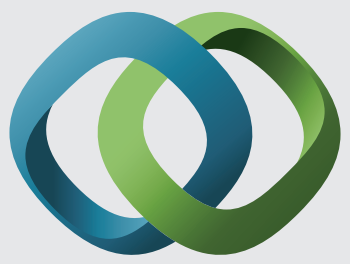

\section{Hindawi}

Submit your manuscripts at

http://www.hindawi.com
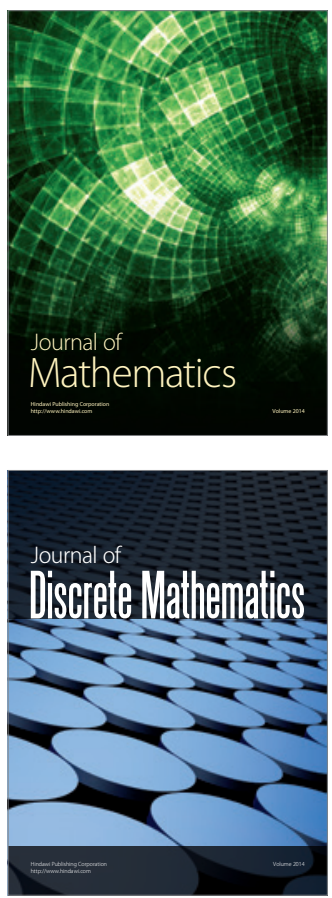

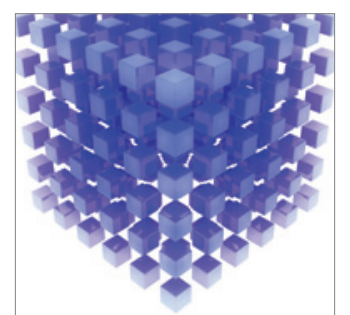

Mathematical Problems in Engineering
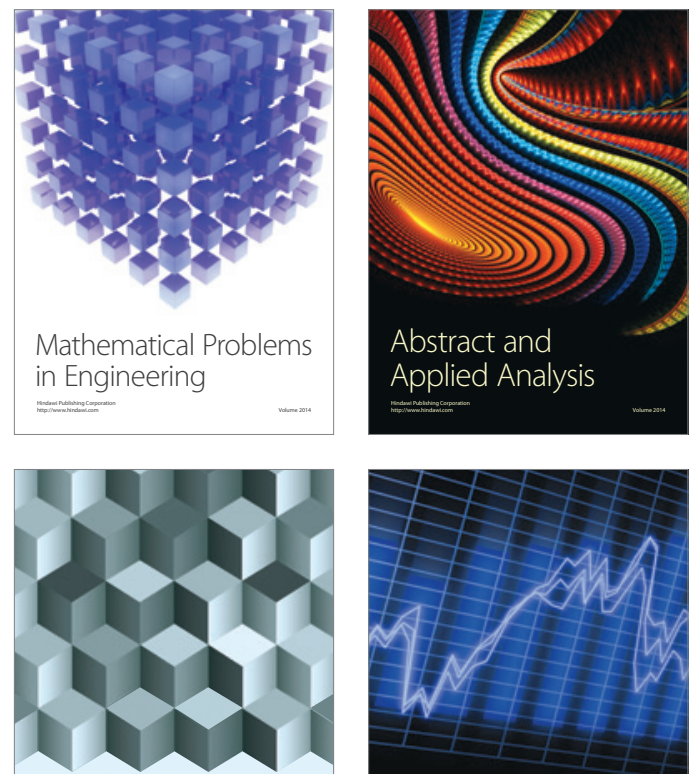

Journal of

Function Spaces

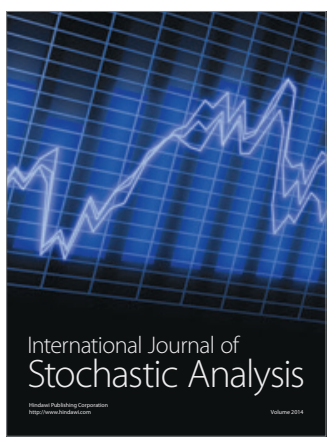

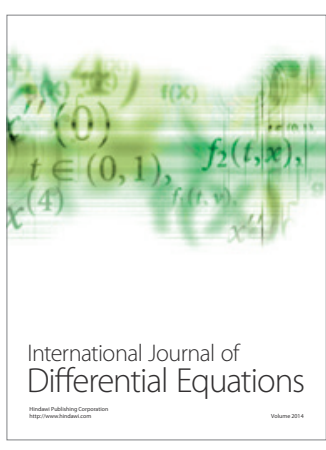
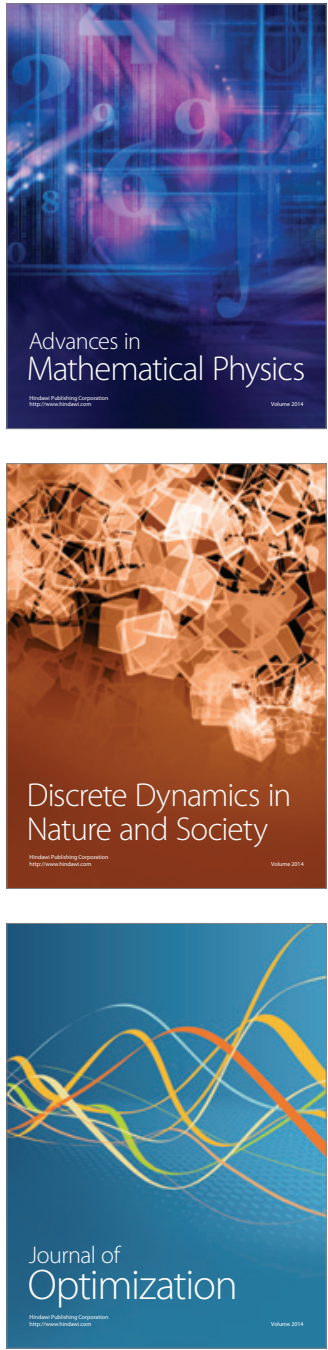